\title{
Image Processing Based Automatic Detection of Asphalt Pavement Rutting Using a Novel Metaheuristic Optimized Machine Learning Approach
}

\section{Minh-Tu Cao}

MUST: Minghsin University of Science and Technology

Kuan-Tsung Chang

MUST: Minghsin University of Science and Technology

Ngoc-Mai Nguyen

National Taiwan University of Science and Technology

Van-Duc Tran

Duy Tan University

Xuan-Linh Tran ( $\nabla$ tranxuanlinh@duytan.edu.vn )

Duy Tan University

Nhat-Duc Hoang

Duy Tan University

\section{Research Article}

Keywords: Rutting Detection, Least Squares Support Vector Machine, Forensic-Based Investigation, Feature Selection, Image Processing.

Posted Date: May 3rd, 2021

DOI: https://doi.org/10.21203/rs.3.rs-334982/v1

License: (c) (1) This work is licensed under a Creative Commons Attribution 4.0 International License. Read Full License

Version of Record: A version of this preprint was published at Soft Computing on August 3rd, 2021. See the published version at https://doi.org/10.1007/s00500-021-06086-5. 


\title{
Image Processing Based Automatic Detection of Asphalt Pavement Rutting Using a Novel Metaheuristic Optimized Machine Learning Approach
}

\section{Title Page}

\author{
Author: $\quad$ Minh-Tu Cao, Ph.D. \\ Position: Assistant Professor \\ Affiliation: Dept. and Ins. of Civil Engineering and Environmental Informatics, Minghsin \\ University of Science and Technology \\ Address: $\quad$ No.1, Xinxing Rd., Xinfeng Hsinchu 30401, Taiwan (R.O.C) \\ Phone: $\quad+886-3-5593142 \# 3289$ \\ Email: $\quad$ caominhtu2201@ mail.must.edu.tw \\ Author: Kuan-Tsung Chang, Ph.D. \\ Position: Associate Professor \\ Affiliation: Dept. and Ins. of Civil Engineering and Environmental Informatics, Minghsin \\ University of Science and Technology \\ Address: $\quad$ No.1, Xinxing Rd., Xinfeng Hsinchu 30401, Taiwan (R.O.C) \\ Phone: $\quad$ +886-3-5593142 \#3281 \\ Email: $\quad$ ktchang@must.edu.tw \\ Author: $\quad$ Ngoc-Mai, Nguyen, M.Sc. \\ Position: Research Asistant \\ Affiliation: Department of Civil and Construction Engineering, National Taiwan \\ University of Science and Technology \\ Address: $\quad$ No.43, Keelung Rd., Sec.4, Da'an Dist., Taipei 10607, Taiwan \\ Phone: $\quad 886227333141$ \\ Email: $\quad$ D10505808@mail.ntust.edu.tw
}

Author: $\quad$ Van-Duc Tran, Ph.D.

Position: Lecturer

Affiliation:

a. International School, Duy tan University, Da Nang, 550000, Vietnam

b. Faculty of Civil Engineering, Duy Tan University, Da Nang, 550000, Vietnam

Address: $\quad$ Danang City, 254 Nguyen Van Linh, Danang 550000, Viet Nam

Phone: $\quad$ (+84) 2036.3827111 (809)

Email: $\quad$ tranvanduc1@dtu.edu.vn

Author: $\quad$ Xuan-Linh Tran, Ph.D. *

Position: Lecturer

Affiliations:

a. Institute of Research and Development, Duy Tan University, Da Nang, 550000, Vietnam

b. Faculty of Civil Engineering, Duy Tan University, Da Nang, 550000, Vietnam

Address: $\quad$ P809 - 03 Quang Trung, Danang, Vietnam

Phone: $\quad$ (+84) $2036.3827111(809)$

Email: $\quad$ tranxuanlinh@duytan.edu.vn 
Author: $\quad$ Nhat-Duc, Hoang, Ph.D.

Position: Lecturer

Affiliations:

a. Institute of Research and Development, Duy Tan University, Da Nang, 550000, Vietnam

b. Faculty of Civil Engineering, Duy Tan University, Da Nang, 550000, Vietnam

Address: $\quad$ P809 - 03 Quang Trung, Danang, Vietnam

Phone: $\quad$ (+84) 2036.3827111 (809)

Email: $\quad$ hoangnhatduc@duytan.edu.vn

*Corresponding author: Xuan-Linh Tran, Institute of Research and Development, Duy Tan University, Da Nang, 550000, Vietnam; Faculty of Civil Engineering, Duy Tan University, Da Nang, 550000, Vietnam; tranxuanlinh@duytan.edu.vn 


\title{
Image Processing Based Automatic Detection of Asphalt Pavement Rutting Using a Novel Metaheuristic Optimized Machine Learning Approach
}

\begin{abstract}
This study presents a novel computer vision based approach to automatically identify rutting appeared on asphalt pavement of road. The developed model is established base on a hybridization of image processing techniques and an advanced machine learning model with support of a metaheuristic optimization engine. Gabor filter and discrete cosine transform are employed to implement context computation for image data, accordingly generate initially extracted features of rutting and non-rutting. Least Squares Support Vector Classification (LSSVC) is then used to learn categorization of rutting and non-rutting based on the extracted features. The final LSSVC prediction model is constructed via a loop of optimization process which is controlled by a novel metaheuristic optimization algorithm, called forensic-based investigation (FBI), to attain optimal model's configuration with ultimate prediction accuracy. This study further utilized a dynamic feature selection (FS) method to integrate in the searching loop to appropriately remove redundant features that provide inconsistent information leading to the compromising of model performance. A dataset of 2000 image samples has been collected during field trip of pavement survey in Da Nang city to form and verify the newly developed model. The statistical results of 20 run times using k-fold cross validation method have demonstrated the hybrid model of FBI-LSSVC-FS to achieve the most desired rutting detection performance with classification accuracy rate, precision, recall, and F1 score of $98.9 \%, 0.994,0.984$ and 0.989 , respectively. Hence, this paper contributes to the core body of knowledge a novel AI-based prediction model to assist transportation agencies in the task of periodic asphalt pavement survey.
\end{abstract}

Keywords: Rutting Detection; Least Squares Support Vector Machine; Forensic-Based Investigation; Feature Selection; Image Processing.

\section{Research Background and Motivation}

The network of asphalt pavement road is definitely the largest component of infrastructure and its essentiality is reflected by the influence on daily activities of people. Therefore, the pavement condition strongly affects the safety of vehicle passengers and any degradations of road surface can bring about multiple negative influences on vehicle passengers as well as economic development of local societies (Gao et al. 2020; Hadjidemetriou et al. 2020; Hadjidemetriou et al. 2018; Liu et al. 2017). Many countries around the globe are currently focusing on maintaining the acceptable road condition. In fact, the funds allocated to rehabilitation activities may significantly surpass those allocated to new road construction projects (Radopoulou and Brilakis 2015).

To cope with an expanding road network and its rapid deteriorating in many road sections, a pavement management system is essential since it provides a systematic approach for establishing cost-effective and timely maintenance and rehabilitation strategies in the whole life cycle of asphalt pavement roads (Yao et al. 2008). Pavement management systems generally demand information of the current pavement condition in order to optimize resource utilization for maintenance and rehabilitation (Eduardo et al. 2014).

The appropriateness of the decision made by a pavement management system strongly depends on pavement condition data collected from periodic pavement survey trips. The current practice of pavement data collection in many developing countries including Vietnam 
is performing visual surveys carried out by human inspectors. Although human visual surveys can help to attain accurate assessments, this conventional practice is notorious for low productivity as well as its strong dependency on the experience and judgment of human inspectors (Guan et al. 2015).

In recent years, scholars and practicing engineers have increasingly investigated alternative solutions for performing the tedious works of periodic pavement condition survey. With the rapid advancements of computer hardware and digital image processing techniques supported by the availability of low-cost digital cameras, automatic pavement assessment has been made feasible and become an effective approach for enhancing the productivity of periodic surveying tasks (Zakeri et al. 2017). The data collected and processed by the image processing based automatic pavement assessment models is particularly useful for transportation authorities since these models provide them with current states of pavement in a timely manner. Based on the collected information, transportation authorities can formulate appropriate plans for repairs and replace activities to optimize their resource allocations. Thus, the chance of road networks disruptions due to incapable serviceability is minimized (Hadjidemetriou et al. 2018).

Based on the recognizable advantages of computer vision-based approaches, various methods for automatic pavement appraisal have been proposed and evaluated recently. Different methods are designed to cope the task of recognizing different pavement defects including cracks (Dorafshan 2017; Dorafshan et al. 2018; Eduardo et al. 2014; Hoang and Nguyen 2018; Hoang et al. 2018b; Kaddah et al. 2018; Li et al. 2017; Ouma and Hahn 2016; Salman et al. 2013; Zhang et al. 2018a), potholes (Akagic et al. 2017; An et al. 2018; Aparna et al. 2019; Hoang 2018; Ouma and Hahn 2017; Yousaf et al. 2018), raveling (Hoang 2019a; Massahi et al. 2018; Mathavan et al. 2014), deformation (Cheng et al. 2018), roughness (Douangphachanh and Oneyama 2014; Georgiou et al. 2018), and patches (Hadjidemetriou et al. 2016; Hadjidemetriou et al. 2018; Hoang 2019b; Radopoulou and Brilakis 2015). Based on the collected information of pavement distresses, transportation authorities can rate the pavement condition and determine appropriate responses to recover deteriorating road sections.

Rutting is also a widely observed type of pavement distresses which critically affects both driving safety and comfort. This type of defect typically occurs when permanent depression accumulates in a pavement surface over time; rutted areas can be recognized by the wheel path imprinted on the road surface (Liley 2018). Severe rutted pavements can lead to traffic accidents due to unevenness of road surface and hydroplaning. Especially for Vietnam where motorcycle is the dominant form of personal transportation means, switching lanes on rutted areas can be detrimental to drivers. Additionally, roughness of rutted pavement may bring out additional vehicle maintenance expense.

While a decent attention has been paid to the task of recognizing cracks, potholes, and patches existing on pavement surface, there are much fewer works dedicating in detecting rutted areas. The detection of rutting is also an essential element for the decision-making process in pavement repair and replacement. Hence, it is a practical need to develop intelligent models capable of detecting rutted areas automatically and accurately. Since rutting belongs to the category of area-based defects, image processing methods for characterizing pavement texture information can be potentially employed.

Kachouie et al. (2004) proposed a hybrid method of a Gabor filter bank and discrete cosine transform to extract features from digital images used for general texture segmentation. Eduardo et al. (2014) constructs a road crack detection model relying on visual features extracted by Gabor filters; the filtered images are then used as descriptors for AdaBoost classifiers. An intelligent model proposed in (Hadjidemetriou et al. 2018) employed discrete cosine transform for automated pavement patch detection. 
Gopalakrishnan et al. (2017) employed deep convolutional neural network and transfer learning for computer vision-based pavement distress recognition. Zhang et al. (2018b) relied on the concept of transfer learning to detect sealed and unsealed pavement cracks. Jo and Jadidi (2019) introduced a crack classification system based on various image filtering techniques and Deep Belief Network. Doycheva et al. (2019) combined computer vision techniques and deep learning models for real-time pavement distress categorization; the authors combined textural features and deep learning to determine the different types of pavement defects including cracks, potholes, and patches.

Based on the reviewed literature, it can be seen that the applications of computer vision for automatic pavement rutting detection are rarely reported. Moreover, following an increasing trend of combining image process and machine learning in the field of automatic pavement survey (Coenen and Golroo 2017; Cubero-Fernandez et al. 2017; Gopalakrishnan 2018; Hoang et al. 2018a; Zakeri et al. 2017; Zhang et al. 2018a), this study aims at filling the gap in the current literature by establishing a hybrid approach of image processing and machine learning approaches for pavement rutting detection. Image processing methods including the discrete cosine transform and Gabor filter are first employed to compute pavement texture. The extracted pavement texture is then used as numerical features and analyzed by a hybrid learning approach of Least Squares Support Vector Classification (LSSVC) (Suykens et al. 2002) and Forensic-Based Investigation (FBI) metaheuristic (Chou and Nguyen 2020).

The LSSVC machine learning approach is employed for constructing a decision boundary which separates rutting samples from non-rutting ones. The role of the FBI algorithm in this study is two-fold. First, the FIB is utilized for automatic feature selection which identifies the most discriminative features extracted by the discrete cosine transform and Gabor filter. Second, this novel metaheuristic algorithm is employed to optimize the LSSVC training phase.

In addition, the integration of image-processing and machine learning approaches for rutting detection has been trained and verified by a set of 2000 pavement image samples and demonstrated promising predictive results. The rest of the paper is organized as follows: The second section reviews the research methodology, followed by the section that describes the image samples; the fourth section presents the proposed model for automatic recognition of asphalt pavement rutting, followed by the next section which reports the experimental results; the last section provides several concluding remarks of this study.

\subsection{Image Processing Techniques}

\section{Research Methodology}

As mentioned earlier, the surface of asphalt pavement is characterized by a diverse form of coarse / fine aggregates, oil/paint stains, traffic marks, and defects such as cracks, potholes, patches, etc. Each of these objects can have distinctive features regarding color and roughness. Therefore, information of color of a certain image region can be helpful to the data classification process. Notably, it is not possible to detect rutting at a pixel level not only because of the sheer number of pixels captured from surveying images, but also because of the fact that color information of a single pixel is definitely not sufficient for rutting recognition. Due to the noisy pavement background and the typical texture of asphalt pavement, two pixels having the same color can actually belong to both rutting and nonrutting pavement areas. To deal with such issue, a large digital image is often separated into a number of non-overlapped image samples (e.g. 32x32 pixels). This separation also helps to accelerate the computational process. Accordingly, the statistical properties of each smallsized image sample can be computed. 


\subsubsection{Discrete Cosine Transform (DCT)}

The DCT is usually chosen because of its fast computation (Tsai et al. 2006). Most approaches to DCT-based texture classification are based on block DCT transformation. The commonly used block size is $16 \times 16$. Then we regard image textures as three one-dimensional vectors characterized by three directional texture properties: vertical, horizontal and diagonal, each of which is located at some specific area of 2-D DCT coefficients. In addition, DC coefficient represents the average energy of an image, which is an important index of an image. Therefore, our proposed feature vector is formed with four major components: the DC coefficient.

The Discrete Cosine Transform (DCT) can be applied to the entire image or to sub-image of various sizes. As described in previous section, most existing work extracts texture features using block-DCT coefficients. In this section, we will describe our approach in this area focusing on using the DCT coefficients transformed from the entire image to generate the global texture feature. The DCT transform for an $N \times N$ image represented by pixel values $f(u, v)$ for $u, v=0,1, \ldots, N-1$ can be defined as (Pun and Zhu 2009; Subudhi and Mukhopadhyay 2017):

$$
C(u, v)=\frac{2}{N} \alpha(u) \alpha(v) \sum_{i=0}^{N-1} \sum_{j=0}^{N-1} f(i, j) \times \cos \left(\frac{(2 i+1) u \pi}{2 N}\right) \times \cos \left(\frac{(2 j+1) v \pi}{2 N}\right)
$$

where $\alpha(x)=1 / \sqrt{2}$ if $x=0$ and 1 if $x \neq 0 ; C(u, v)$ is the DCT coefficient.

The contribution of this work lies in generating the dominant features of global image texture. The DCT method can be used to generate feature from digital images because the DC coefficient $\mathrm{C}(0,0)$ represents the average energy of the image. Moreover, all the remaining coefficients contain frequency information which produces a different pattern of image variation. The coefficients of some regions represent some directional information (Hee-Jung and Sung-Hwan 1997; Tsai et al. 2006). The extracted DCT coefficients used for texture representations are illustrated in Fig. 1. The DC coefficient $\mathrm{C}(0,0)$ as well the coefficients characterizing vertical texture region, horizontal texture region, and diagonal texture region are computed. In total, there are 30 DCT based texture descriptors can be obtained from one image sample (Tsai et al. 2006).

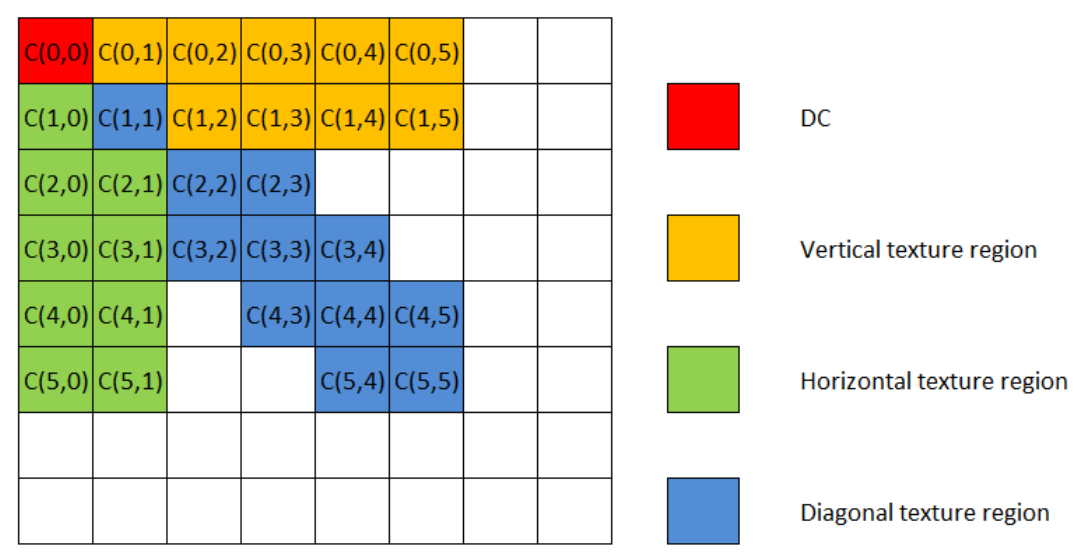

Fig. 1 The extracted DCT coefficients

\subsubsection{Gabor Filter (GF)}

Gabor filtering has optimal joint localization in both spatial and spatial-frequency domains and has been used to detect defects in highly regular textured surfaces (Eduardo et al. 2014). These filters have been widely applied for various tasks including texture segmentation (Kachouie et al. 2004), surface defect detection (Medina et al. 2011), vehicle detection 
(Zehang et al. 2005), texture classification (Kim and So 2018), etc. In essence, a twodimensional Gabor filter is a complex sinusoidal wave modulated by a Gaussian envelope (Hammouda 2000). The filter performs a localized and oriented frequency analysis of a twodimensional signal. The response of a Gabor filter can be mathematically expressed as follows (Jain and Farrokhnia 1991):

$$
h(x, \mathrm{y})=\exp \left\{-\frac{1}{2}\left[\frac{x^{2}}{\sigma_{x}^{2}}+\frac{y^{2}}{\sigma_{y}^{2}}\right]\right\} \cos \left(2 \pi \mathrm{u}_{0} \mathrm{x}\right)
$$

where $u_{0}$ is the frequency of a sinusoidal plane wave along the $x$-axis. $\sigma_{x}$ and $\sigma_{y}$ are the space constants of the Gaussian envelope along the $\mathrm{x}$ and $\mathrm{y}$ axes, respectively. Filters with arbitrary orientations can be obtained via a rigid rotation of the $x-y$ coordinate system.

The frequency domain representation of a Gabor filter can be described as followed (Jain and Farrokhnia 1991):

$$
H(u, v)=A\left(\exp \left\{-\frac{1}{2}\left[\frac{\left(u-u_{0}\right)^{2}}{\sigma_{u}^{2}}\right]+\frac{v^{2}}{\sigma_{v}^{2}}\right\}+\exp \left\{-\frac{1}{2}\left[\frac{\left(u+u_{0}\right)^{2}}{\sigma_{u}^{2}}\right]+\frac{v^{2}}{\sigma_{v}^{2}}\right\}\right)
$$

where $\sigma_{u}=\frac{1}{2} \pi \sigma_{x}, \sigma_{v}=\frac{1}{2} \pi \sigma_{y}$, and $A=2 \pi \sigma_{x} \sigma_{y}$.

To implement Gabor filters, it is required to specify their tuning parameters including the orientation angles and the radial frequency. As recommended by Jain and Farrokhnia (1991), four values of orientations are used: $0^{\circ}, 45^{\circ}, 90^{\circ}$, and $135^{\circ}$. For an image with a width of $N_{w}$ pixels and $N_{w}$ is a power of 2 , the following values of radial frequency $u_{0}$ are used: $1 \sqrt{2}, 2 \sqrt{2}, 4 \sqrt{2}, \ldots, \frac{N_{w}}{4} \sqrt{2}$. Accordingly, statistical measurements of the Gabor filter based image can be computed and used as features for texture classification (Jo and Jadidi 2019).

\subsection{Machine Learning and Metaheuristic Methods}

\subsubsection{The Forensic-Based Investigation (FBI)}

The FBI algorithm, first proposed in (Chou and Nguyen 2020), is inspired by the forensic investigation process of police officers, specifically the criminal investigation process. As soon as a criminal activity is found reported, polices starts an investigation process to catch the suspect. The most wanted suspect has the same character as the optimal solution while police officers act based on sharing and receiving analyzed information to reach the location of the most wanted suspect.

The investigation area of the investigation process is represented by the boundary of search algorithm. The case is closed, meaning that the searching process of algorithm is terminated. Fig. 2 present flowchart of the FBI algorithm following the concept of a common investigation process. The FBI algorithm is composed of two phases: phase A presents the actions of investigation team; and phase B is performed by pursuit team with police agent members.

Step A1: with the trace collection of the current locations and information shared from other locations, investigator can have more basis to infer a new investigation location $A_{i j}^{\prime}$ from the suspected location $A_{i j}$. The transit of suspected locations is given by the Eq. (4). The trace collected at the newly inferred location is then evaluated and compared with that of the old location to determine whether shifting the investigation concentration on the new location or retaining the current location.

$$
A_{i j}^{\prime}=A_{i j}+\operatorname{rand} *\left(A_{i j}-\frac{A_{k j}+A_{h j}}{2}\right)
$$


where $k, h$, and $i$ are three different suspected locations: $\{k, h, i\} \in\{1,2, \ldots, N P\}, j=1,2, \ldots$, $D$; NP is the number of suspected locations; $D$ is the number of dimensions; and rand is a random number in the range $[-1,1]$.

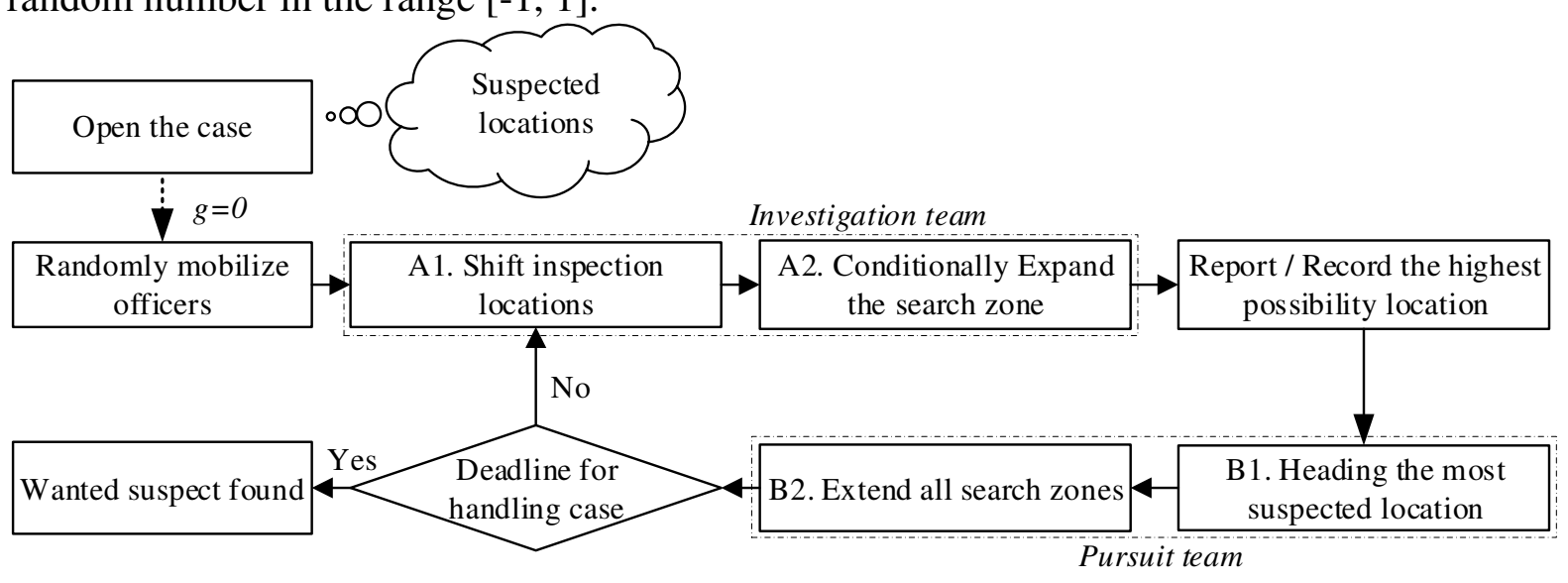

Fig. 2 Flowchart of the FBI algorithm

Step A2: suspected locations with more traces collected will be further searched which is selected depending on the quality of collected locations. Hence, the suspected location with the lower probability $\left(\boldsymbol{P} \boldsymbol{r} \boldsymbol{O}\left(\boldsymbol{A}_{\boldsymbol{i j}}\right)\right.$ has more chance to be updated. The chance of further search is regarded as a probability value calculated as Eq. (5). It is noted that the directions of further investigation are randomly selected in order to increase the diversity of search area. The new location in this context is determined as Eq. (6):

$$
\begin{aligned}
& \operatorname{Pro}\left(A_{i j}\right)=1-\left(p_{A_{i}}-p_{m i n}\right) /\left(p_{m a x}-p_{m i n}\right) \\
& A_{i j}^{\prime}=A B_{b e s t}+A_{d j}+\operatorname{rand}\left(A_{k j}-A_{h j}\right)
\end{aligned}
$$

where $A B_{\text {best }}$ is the location with the highest possibility; $A_{i j}^{\prime}$ is the new suspected location of $A_{i j}$. rand is the random number in the range [-1, 1]; and $d, k, h$, and $i$ are four different suspected locations: $\{d, k, h, i\} \in\{1,2, \ldots, N P\}$.

Step B1: after all the suspected locations are evaluated and delineated, the pursuit team with agent members are deployed to approach instantly. It is worth noting that there is a slight difference between the approach position and the center of the delineated area due to the effect of the information received from the suspected location with the highest possibility. Hence, the newly approached position is determined as Eq. (7) as follows:

$B_{i j}^{\prime}=\operatorname{rand} * B_{i j}+\operatorname{rand} *\left(B A_{b e s t}-B_{i j}\right)$

where $B A_{\text {best }}$ is the located of the highest possibility given by the investigation team; $\operatorname{rand}(s)$ are two random numbers in the range $[0,1] ; j=1,2, \ldots, D$.

Step B2: the police agents collect trace and send basic assessment to the headquarter at every move. The headquarter updates and uses valuable information to evaluate and request the police agents to further expand the searching beyond the delineated area if necessary. New locations rely on the probability value $p_{B_{i}}$ which is compared with a threshold value $p_{B_{r}}$. The new location is calculated as Eq. (8) or Eq. (9) updated if it provides more information of suspect. The location with the most valuable trace found is then transferred to the investigation team for analysis.

$B_{i}^{\prime}=B_{r}+\operatorname{rand} *\left(B_{r}-B_{i}\right)+\operatorname{rand}\left(B A_{b e s t}-B_{r}\right) \quad$ if $p_{B_{r}}>p_{B_{r}}$

$B_{i}^{\prime}=B_{i}+\operatorname{rand} *\left(B_{i}-B_{r}\right)+\operatorname{rand}\left(B A_{b e s t}-B_{i}\right) \quad$ if $p_{B_{r}} \leq p_{B_{r}}$

where $A B_{b e s t}$ is the updated position of the highest possibility, $\operatorname{rand}(s)$ are two random numbers in the range $[0,1] ; B_{r}$ is a randomly selected police agent; $j=1,2, \ldots, D$. 


\subsubsection{Least Squares Support Vector Classification (LSSVC)}

In order to address the problem of large computational complexity of support vector machine, Suykens et al. (2002) proposed to integrate the least square method with an assumption that the dataset $S=\left\{\left(x_{i}, y_{i}\right)\right\}$ can be presented by a non-linear function as shown in Eq. (10), where $\left(x_{i}, y_{i}\right)$ is the $i^{\text {th }}$ data pattern of the training dataset; $y_{i} \in\{-1,1\}$ is for two class labels of interest. Accordingly, a LSSVC model is established by constructing a hyperplane to separating differently categorized data.

$y(x)=\omega^{T} \phi(x)+b$

where $x \in R^{n}, y \in R$, and $\phi(x): R^{n} \rightarrow R^{n h}$ is the transfer to the high dimensional feature space, $b$ is the bias term.

A constrained optimization function $\mathrm{R}$ is introduced to guide the separating hyperplane which is comprised of a sum of squared fitting error $\left(\varepsilon_{i}\right)$ and a ridge regression. Ridge regression is multiplied with regulation coefficient $(\mathrm{C})$ to adjust weights of two components in the equation as shown in Eq. (11).

Minimize: $R(\omega, \varepsilon, b)=\frac{1}{2}\|\omega\|^{2}+C \sum_{i=1}^{n} \varepsilon_{i}^{2}$

Subjected to: $y_{i}=\omega^{T} \phi\left(x_{i}\right)+b+\varepsilon_{i}, i=1, \ldots, n$

The Lagrangian function $L$ is then introduced to handle the unsolvable of $R$ function when when $\omega$ is infinite.

$L(\omega, b, \varepsilon, \xi)=\frac{1}{2}\|\omega\|^{2}+C \sum_{i=1}^{n} \varepsilon_{i}^{2}-\sum_{i=1}^{n} \xi_{i}\left(\omega^{T} \phi\left(x_{i}\right)+b+\varepsilon_{i}-y_{i}\right)$

where $\xi_{k}$ is Lagrangian multiplier. The conditions for optimality are given by:

$\left\{\begin{array}{l}\frac{\partial L}{\partial \omega}=0 \rightarrow \omega=\sum_{i=1}^{n} \xi_{k} \phi\left(x_{k}\right) \\ \frac{\partial L}{\partial b}=0 \rightarrow \sum_{i=1}^{n} \xi_{k}=0 \\ \frac{\partial L}{\partial \varepsilon_{i}}=0 \rightarrow \xi_{i}=C \varepsilon_{i}, i=1, \ldots, n \\ \frac{\partial L}{\partial \xi_{i}}=0 \rightarrow \omega^{T} \phi\left(x_{k}\right)+b+\varepsilon_{i}-y_{i}=0, i=1, \ldots, n\end{array}\right.$

By excluding $\varepsilon$ and, a linear system is returned as Eq. (14):

$\left[\begin{array}{cc}0 & e^{T} \\ e & K+1 / C\end{array}\right]\left[\begin{array}{l}b \\ \xi\end{array}\right]=\left[\begin{array}{l}0 \\ y\end{array}\right]$

where $y=y_{1}, \ldots, y_{n}, 1_{v}=[1 ; \ldots ; 1]$; and $\xi=\left[\xi_{1} ; \ldots ; \xi_{n}\right]$ and $e=[1, \ldots, 1]^{T}$. The kernel function is as follows:

$K\left(x_{i}, x_{k}\right)=k\left(x_{i}, x_{j}\right)=\phi\left(x_{i}\right)^{T} \phi\left(x_{k}\right)$

The output value of the resulting LSVC model is formed as Eq. (16), as follows:

$y(x)=\operatorname{sign}\left(\sum_{i=1}^{n} \xi_{i} K\left(x_{i}, x_{k}\right)+b\right)$

$K\left(x_{i}, x_{k}\right)=\exp \left(-\frac{\left\|x_{i}-x_{k}\right\|^{2}}{2 \gamma^{2}}\right)$

(17)

where $\xi_{k}$ are the solution to the linear system; $\gamma$ is a parameter of kernel function.

This study purposely used the radial basis function (RBF) as kernel function as shown in Eq. (17) since its efficacy has been endorsed in literature studies. Hence, in addition to regularization parameter $(C)$, users are required to properly set value of kernel parameter $(\gamma)$ which controls the smoothness of separating hyperplane, presenting the influencing of the support vectors to the data points. to attains the ultimate generalizability of the LSSVC model (Wu and Shen 2018). Hence, the current study thus employs FBI search engine to identify the optimal values of $C$ and $\gamma$. 


\section{The Collection of Pavement Image Samples}

As stated earlier, the employed machine learning model of the LSSVC is a supervised machine learning approach. Thus, to establish a pavement rutting detection model, a set of 2000 pavement images with the ground truth label has been collected. The numbers of image samples in the positive class (rutting) and negative class (non-rutting) are both 1000 to achieve a balanced classification. Moreover, the digital image samples are collected during field trip of pavement survey in Da Nang city (Vietnam). Image samples of the two categories of non-rutting (class label $=0$ ) and rutting (class label $=1$ ) has been prepared for model training and testing. To facilitate the speed of the feature extraction phase, the DCT computation, and to guarantee the consistency of an image region, the size of image sample has been set to be $32 \times 32$ pixels.

The collected image samples are demonstrated in Fig. 3. It is also noted that the ground truth class label of each image is determined by human inspectors. Furthermore, to mimic the diversity of real-world pavement surface, the negative class of non-rutting consists of samples of intact pavement, cracks, stains, raveling, patch, and traffic marks. The positive class of rutting includes rutting areas with diversiform texture. The image samples have been captured by the Cannon EOS M10 (CMOS 18.0 MP) and Nikon D5100 (CMOS 16.2 MP).
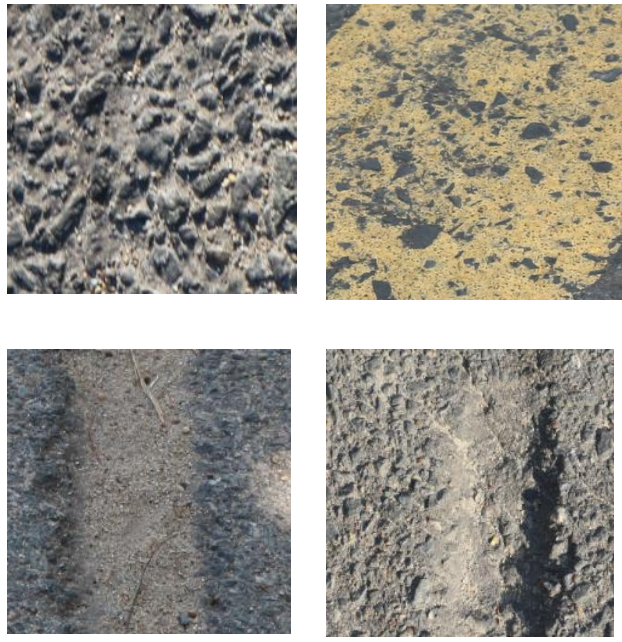

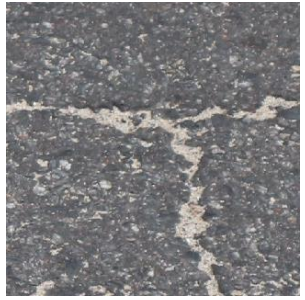

(a)

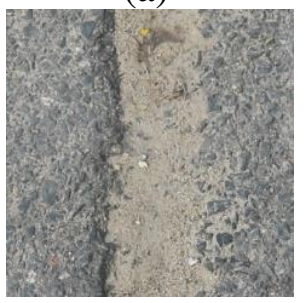

(b)
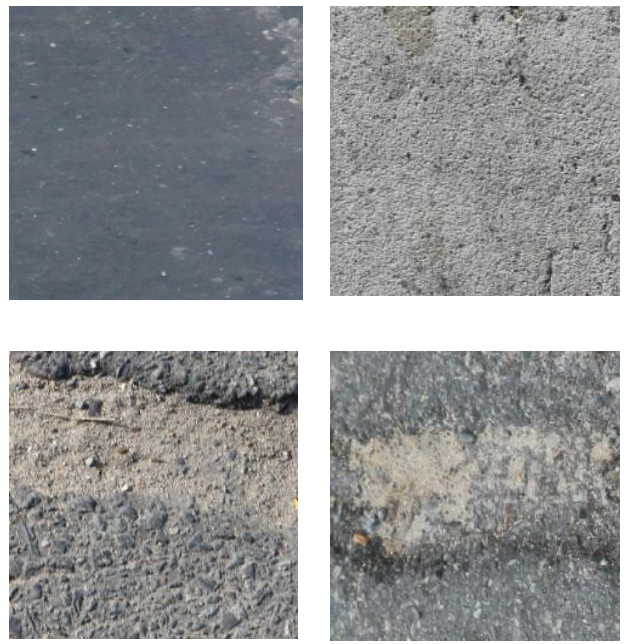

Fig. 1 Illustration of the collected pavement images: (a) Non-rutting class and (b) Rutting class

\section{The Proposed Hybridization of Image Processing, FBI, and LSSVC for Automatic Detection of Asphalt Pavement Rutting}

The proposed hybrid framework of image processing, FBI, and LSSVC for detection of asphalt pavement rutting, denoted as FBI-LSSVC-FS, consists of three main steps: (i) image texture computation, (ii) FBI based model optimization, and (iii) data classification. The general structure of the FBI-LSSVC-FS's model optimization is demonstrated in Fig. 4. The first step computes texture information of pavement surface obtained from 2000 image samples. The second step processes the texture information; the FBI is used to reduce the dimensionality of the original data which facilitates subsequent data classification phase. In the final step, the LSSVC optimized by the FBI model is employed to establish a decision surface that separates the input space into two domains of rutting and non-rutting. The FBILSSVC-FS model has been developed in MATLAB environment and tested in the HP Z440 Workstation (Intel (R) Xeon (R) CPU E5-1630 v3 @ 3.7GHz, RAM 16GB). In addition, the 
Gabor filter and the individual decision tree model are constructed with the help of the Accord.NET Framework (Accord 2019).

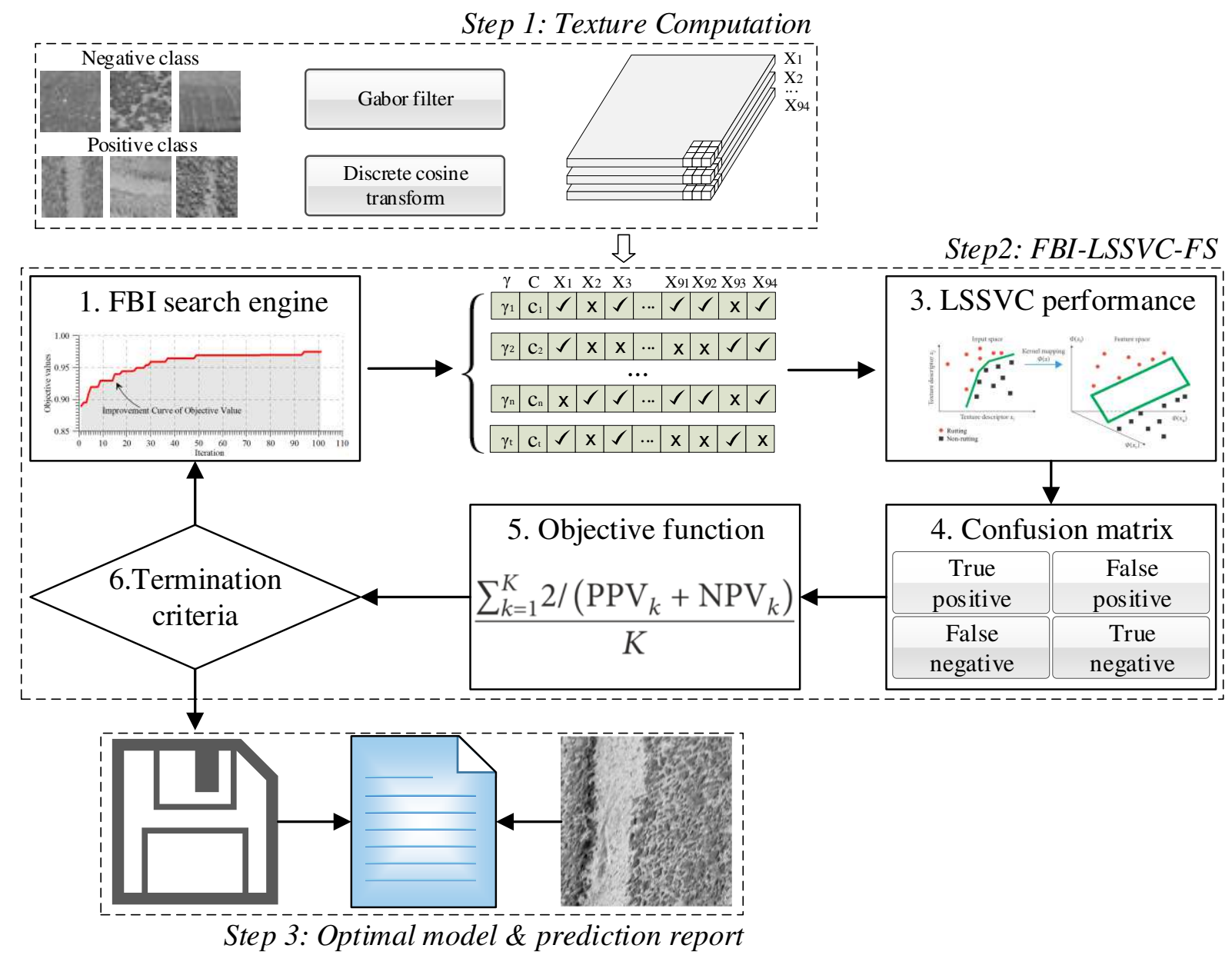

Fig. 4 The proposed FBI-LSSVC-FS Model Structure

\subsection{Image Feature Extraction}

As mentioned earlier, image samples in this study are processed by the DCT and GF techniques to extract numerical features used for rutting detection. Demonstrations of the image processing phases are provided in Fig. 5. It is noted that prior to the texture computation process, the original image samples have been converted to gray-scale images. As suggested by Jain and Farrokhnia (1991), four values of orientations $\left(0^{\circ}, 45^{\circ}, 90^{\circ}\right.$, and $\left.135^{\circ}\right)$ and four values of radial frequency $(1 \sqrt{2}, 2 \sqrt{2}, 4 \sqrt{2}, 8 \sqrt{2})$ are used to characterize image texture obtained from the employed Gabor filters. Accordingly, statistical measurements of the Gabor filters are computed and employed as texture-based features. The statistical measurements include the mean, standard deviation, skewness, and entropy which are computed as follows.

The mean of the Gabor filter response is given by:

Mean $_{\text {GaborFilter }}=\frac{\sum_{i=0}^{H-1} \sum_{j=0}^{W-1} \operatorname{GFR}(\mathrm{i}, \mathrm{j})}{W \times H}$

where $H$ and $W$ are the width and height of an image sample, respectively. GFR $(i, j)$ denotes the Gabor filter response at an pixel $(i, j)$. 
The standard deviation of the Gabor filter response is computed as follows:

$S T D_{\text {GaborFilter }}=\frac{\sum_{i=0}^{H-1} \sum_{j=0}^{W-1}\left[G F R(\mathrm{i}, \mathrm{j})-\mathrm{Mean}_{\mathrm{GaborFilter}}\right]^{2}}{W \times H}$

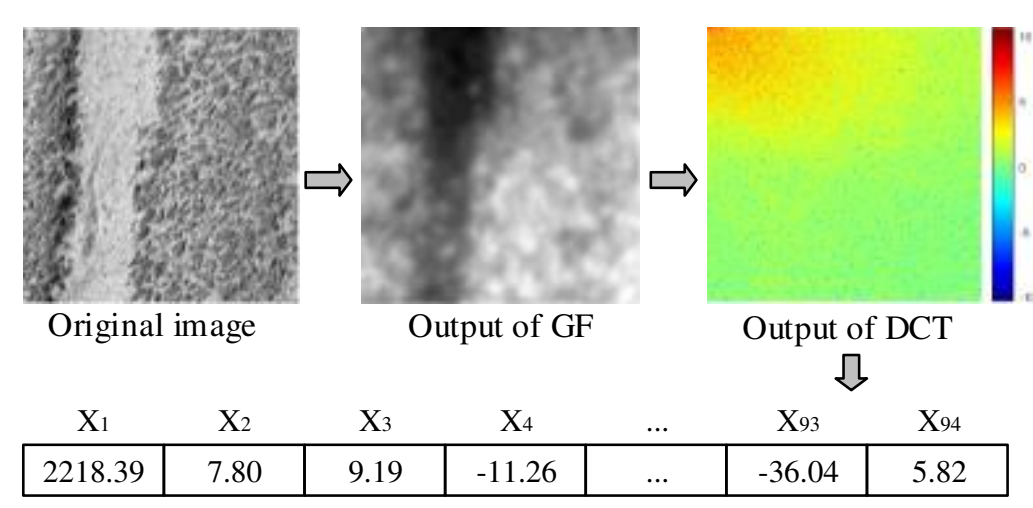

Fig. 5 Illustration of the feature extraction process

The equation used to calculate the skewness of the Gabor filter response is given by (Joanes and Gill 1998):

Skewness $_{\text {GaborFilter }}=\frac{\frac{1}{W \times H} \sum_{i=0}^{H-1} \sum_{j=0}^{W-1}\left[\operatorname{GFR}(\mathrm{i}, \mathrm{j})-\text { Mean }_{\text {GaborFilter }}\right]^{3}}{\left[\frac{1}{W \times H-1} \sum_{i=0}^{H-1} \sum_{j=0}^{W-1}\left\{G F R(\mathrm{i}, \mathrm{j})-\text { Mean }_{\text {GaborFilter }}\right\}^{2}\right]^{3 / 2}}$

The entropy of the Gabor filter response is given by:

Entropy $_{\text {GaborFilter }}=-\sum_{i=0}^{N D V-1} P_{\text {Filter }} \times \log _{2}\left[P_{\text {Filter }}\right]$

where $P_{\text {Filter }}$ denotes the first-order histogram of the Gabor filter response. $N D V=256$ represents the number of discrete intensity values.

Since there are 16 Gabor filters and each filter results in four statistical measurements (the mean, standard deviation, skewness, and entropy), the number of Gabor filter-based features is 64. As explained earlier, the number DCT based texture descriptors is 30. Moreover, the used DCT block size is $16 \times 16$. Therefore, the total number of texture-based features is 94 .

\subsection{Model Optimization based on the FBI metaheuristic}

It is noted that all of the features extracted from step I with support of GF and CDT techniques are then fed to the step II for establishing the rutting detection model. Since the scale values of extracted features are broadly different, they need to be standardized to avoid negative impact of features with large values against the others. Hence, this study used the Zscore equation to normalize all feature values since this type of data transformation is highly perceived for classification problems. The Z-score equation is given by:

$X_{Z N}=\frac{X_{o}-m_{X}}{s_{X}}$

where $X_{o}$ and $X_{Z N}$ denotes an original and a normalized data, respectively. $m_{X}$ and $s_{X}$ represent the mean and the standard deviation of the original data, respectively. 
Subsequently, concerned parameters are randomly generated initial values to trigger the commencement of the FBI-based searching loop. As FBI simultaneously determines the best pair of LSSVC's hyper-parameter values $(\gamma$ and $C$ ) and set of high influencing predictors (features), a total of 96 variables necessities to be taken into account in which variables for deciding the present of features to are binary ( 0 or 1$)$. As seen in Table 1, this study set population size of the FBI is as 200. The population size is thus roughly twice as many as searching dimensions while iteration of searching loop is set to be 100. Initial boundaries of $\gamma$ and $\mathrm{C}$ are set in the ranges $10^{-6}$ and $10^{6}$, respectively.

Accordingly, the LSSVC obtains various sets of hyper-parameter values to build the classification model of rutting / non-rutting. It is noted that the LSSVC only uses the training dataset while testing dataset is reserved for testing the prediction power of the FBI-optimized model. This study employed a $k$-fold validation method with $k=5$ to avoid the bias of data partition for training LSSVC. Therefore, the training dataset is partitioned into 5 exclusively mutual subsets. Accordingly, LSSVC is trained repeatedly 5 times for each set of parameter values in which 4 folds are merged for use of training model while the remaining fold is served for validation. The outcomes of this step are images labeled which are then compared with their true class in the confusion matrix step.

An objective function is formulated by averaging sum of positive predictive values (PPV) and negative predictive values (NPV) for evaluating quality of FBI-provided solutions, shown in Eq. (23). $K=5$, stands for number of folds. PPV and NPV are utilized to express the model performance associated with a set of LSSVC hyper-parameters and selected features. Further, use of both PPV and NPV prevents the prediction model from being in favor of a certain class. PPV and NPV are calculated as Eq. (24) and Eq. (25), respectively.

$$
\begin{aligned}
& f=\frac{1 / 2 * \sum_{i}^{K}\left(P P V_{i}+N P V_{i}\right)}{5} \\
& P P V=\frac{T P}{T P+F P} \\
& N P V=\frac{T N}{T N+F N}
\end{aligned}
$$

where: where TP, TN, FP, and FN are the true positive, true negative, false positive, and false negative values, respectively.

The FBI will employ values of the objective function as the basis to adjust solutions in accordance with its working procedure. The FBI retains better solutions and abandons poor solutions during its searching process. Hence, the values of the objective functions will be improved gradually until they reach the most desired values or stopping condition is met. Once the searching terminates, the optimized LSSVC is ready for predicting new image data of rutting / non-rutting.

Table 1 Initial control parameter settings

\begin{tabular}{llccc}
\hline \multicolumn{1}{c}{ Model Member } & \multicolumn{1}{c}{ Tuning parameter } & Notation & Lower bound & Upper bound \\
\hline \multirow{2}{*}{ LSSVC Model } & Gama & $\gamma$ & $10^{-6}$ & $10^{6}$ \\
& Regularization coefficient & $C$ & $10^{-6}$ & $10^{6}$ \\
\hline \multirow{2}{*}{ FBI search engine } & Iteration number & $I_{\text {ter }}$ & & 100 \\
\cline { 2 - 4 } & Population size & Pop & & 100 \\
\hline 94 Extracted Features & & $F S$ & 0 & 1 \\
\hline
\end{tabular}

Noted: Variable for selection / de-selection offeatures is binary: 0 or 1 


\section{Experimental Result and Comparison \\ 5.1 Experimental Result Comparison and Discussion}

This paper analyzed the predictive capability of the FBI-LSSVC-FS based on statistical results of 20 run times which is large enough to accurately measure the reliability of a classification model. In order to avoid the bias in the data partition process, the crossvalidation approach should be used for partitioning dataset (Orenstein et al. 1995). Thus, all of the data patterns will be ascertained to involve in both training and testing phase. Accordingly, the training and testing sets account for $90 \%$ and $10 \%$ of the data size, respectively. Hence, the analysis is conducted on the average value and standard deviation value obtained from 20 run times. Fig. 6 demonstrates the improvement of objective function's value for a certain run time.

To highlight the prediction capability of the FBI-LSSVC-FS model, its performance has been compared to that of FBI-LSSVC (without feature selection), LSSVC, Random forest (RF), and General regression neural network (GRNN), and SVC. The FBI-LSSVC and LSSVC models are selected to interpret the importance of each technique integrated in the proposed road rutting classification model. GRNN, SVC, and RF represents for different types of classification framework which also highly perceived by scholars in may real application. Hence, inclusion of those techniques for comparing models' performance is essential. All but FBI-LSSVC-FS and FBI-LSSVC models are manually implemented by trial-and-error method to possibly tune the good parameter values.

All soft computing models are performed 20 times with the corresponding training and testing datasets as FBI-LSSVC-FS model. The performance of all models is compared on variety of evaluation criteria, including: classification accuracy rate (CAR), sensitivity (recall), precision or positive predictive value (PPV), negative predictive value (NPV), and F1score. The use of many evaluation criteria aims at giving the correct assessment for all aspects. Thus, models' weakness in classifying data samples will be exposed. Since the PPV and NPV have been introduced earlier, the CAR, Recall, and F1 Score measurement metrics are computed as follows:

$$
\begin{aligned}
& \text { CAR }=\frac{T P+T N}{T P+T N+F P+F N} \times 100 \% \\
& \text { Recall }=\frac{T P}{T P+F N}
\end{aligned}
$$

where $T P$ is number of truly predicted rutting damages; $T N$ is number of truly predicted nonrutting damages; $F P$ is number of wrongly predicted rutting damages; and $F N$ is number of wrongly predicted non-rutting damages. 


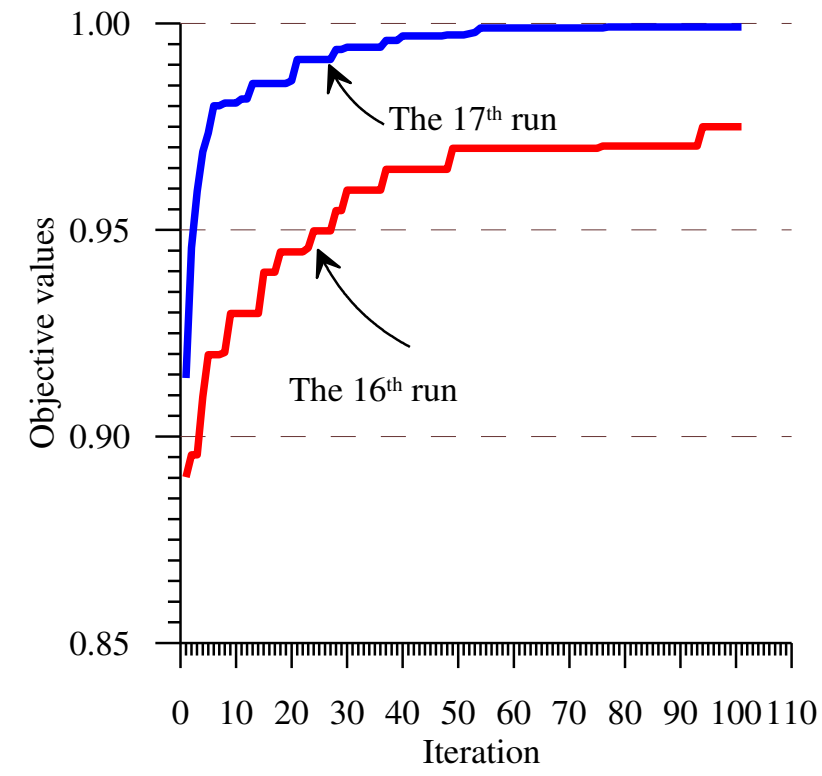

Fig. 6 Improvement of objective values

Table 2 Output of confusion matrix for 20 run times

\begin{tabular}{|c|c|c|c|c|c|c|}
\hline Run no. & $\mathrm{TP}$ & $\mathrm{TN}$ & FP & FN & $\begin{array}{c}\text { Features } \\
\text { used }\end{array}$ & $\begin{array}{l}\text { Features } \\
\text { excluded }\end{array}$ \\
\hline Run 1 & 104 & 92 & 3 & 1 & 57 & 37 \\
\hline Run 2 & 95 & 101 & 0 & 4 & 55 & 39 \\
\hline Run 3 & 94 & 102 & 1 & 3 & 51 & 43 \\
\hline Run 4 & 104 & 94 & 0 & 2 & 49 & 45 \\
\hline Run 5 & 99 & 101 & 0 & 0 & 46 & 48 \\
\hline Run 6 & 92 & 103 & 1 & 4 & 51 & 43 \\
\hline Run 7 & 94 & 105 & 0 & 1 & 61 & 33 \\
\hline Run 8 & 100 & 100 & 0 & 0 & 53 & 41 \\
\hline Run 9 & 102 & 95 & 1 & 2 & 60 & 34 \\
\hline Run 10 & 99 & 99 & 2 & 0 & 45 & 49 \\
\hline Run 11 & 112 & 88 & 0 & 0 & 44 & 50 \\
\hline Run 12 & 89 & 111 & 0 & 0 & 55 & 39 \\
\hline Run 13 & 99 & 101 & 0 & 0 & 58 & 36 \\
\hline Run 14 & 86 & 112 & 1 & 1 & 31 & 63 \\
\hline Run 15 & 94 & 102 & 0 & 4 & 56 & 38 \\
\hline Run 16 & 100 & 96 & 2 & 2 & 46 & 48 \\
\hline Run 17 & 100 & 95 & 0 & 5 & 42 & 52 \\
\hline Run 18 & 102 & 98 & 0 & 0 & 53 & 41 \\
\hline Run 19 & 101 & 97 & 1 & 1 & 50 & 44 \\
\hline Run 20 & 103 & 95 & 1 & 1 & 55 & 39 \\
\hline
\end{tabular}

Noted: $T P=$ true positive $; N=$ true negative; $F P=$ false positive $F N=$ false negative 


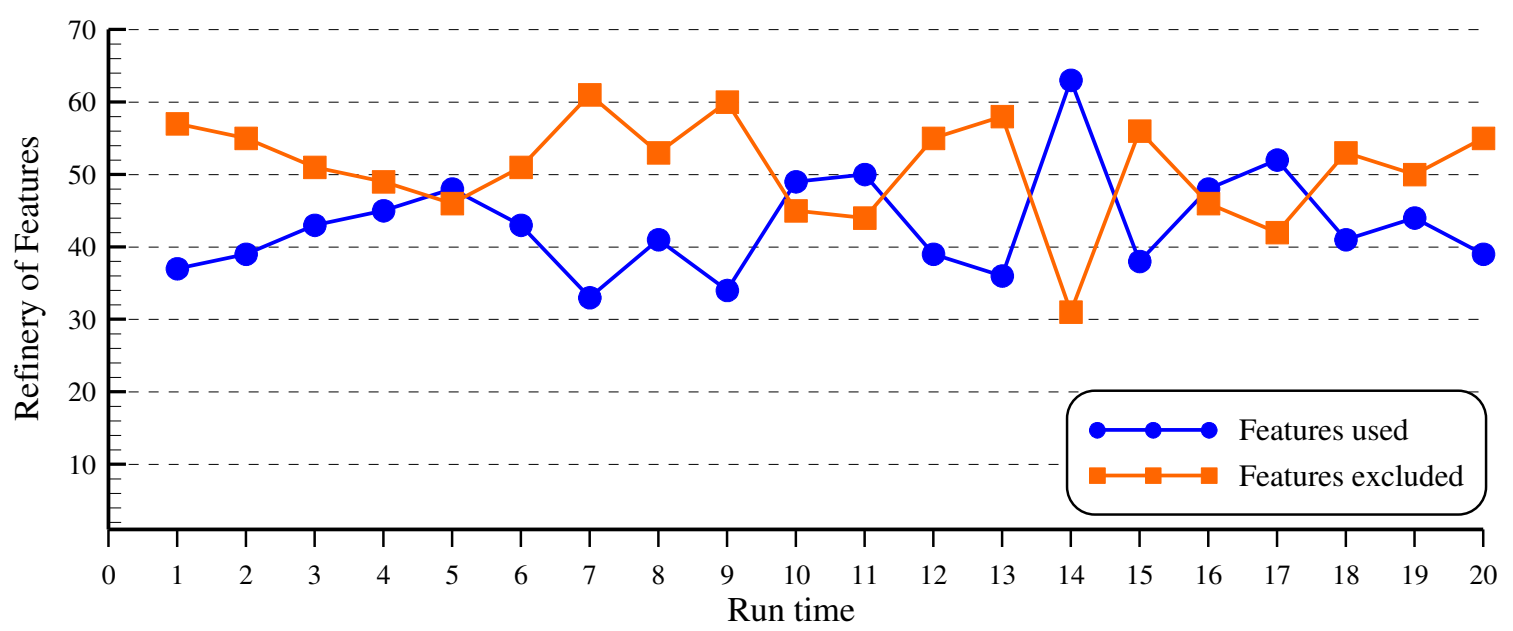

Fig. 7 Features refined for LSSVC model construction

Table 3 Summary of comparative models' performance

\begin{tabular}{|c|c|c|c|c|c|c|c|}
\hline & Model & $\mathrm{RF}$ & GRNN & SVC & LSSVC & FBI-LSSVC & FBI-LSSVC-FS \\
\hline \multirow{5}{*}{ 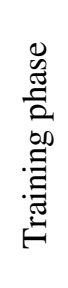 } & CAR & $99.9 \% \pm 0.1 \%$ & $100 \% \pm 0.0 \%$ & $96.5 \% \pm 0.3 \%$ & $92.0 \% \pm 0.3 \%$ & $98.5 \% \pm 1.0 \%$ & $99.4 \% \pm 0.5 \%$ \\
\hline & Prec. & $0.999 \pm 0.001$ & $1.000 \pm 0.000$ & $0.966 \pm 0.003$ & $0.909 \pm 0.003$ & $0.987 \pm 0.013$ & $0.998 \pm 0.003$ \\
\hline & Recall & $0.998 \pm 0.002$ & $1.000 \pm 0.000$ & $0.965 \pm 0.003$ & $0.934 \pm 0.003$ & $0.980 \pm 0.013$ & $0.989 \pm 0.007$ \\
\hline & F1_score & $0.999 \pm 0.001$ & $1.000 \pm 0.000$ & $0.965 \pm 0.003$ & $0.921 \pm 0.002$ & $0.984 \pm 0.012$ & $0.994 \pm 0.007$ \\
\hline & NPV & $0.998 \pm 0.002$ & $1.000 \pm 0.000$ & $0.965 \pm 0.003$ & $0.932 \pm 0.003$ & $0.980 \pm 0.012$ & $0.989 \pm 0.005$ \\
\hline \multirow{5}{*}{ 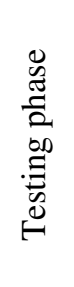 } & CAR & $93.0 \% \pm 1.9 \%$ & $91.8 \% \pm 2.1 \%$ & $92.5 \% \pm 1.9 \%$ & $90.6 \% \pm 2.0 \%$ & $95.3 \% \pm 1.3 \%$ & $98.9 \% \pm 0.9 \%$ \\
\hline & Prec. & $0.932 \pm 0.024$ & $0.911 \pm 0.027$ & $0.920 \pm 0.022$ & $0.894 \pm 0.029$ & $0.949 \pm 0.017$ & $0.994 \pm 0.008$ \\
\hline & Recall & $0.930 \pm 0.035$ & $0.925 \pm 0.029$ & $0.930 \pm 0.029$ & $0.922 \pm 0.027$ & $0.956 \pm 0.021$ & $0.984 \pm 0.016$ \\
\hline & F1_score & $0.931 \pm 0.024$ & $0.918 \pm 0.022$ & $0.925 \pm 0.019$ & $0.907 \pm 0.018$ & $0.952 \pm 0.020$ & $0.989 \pm 0.016$ \\
\hline & NPV & $0.931 \pm 0.034$ & $0.924 \pm 0.029$ & $0.929 \pm 0.029$ & $0.920 \pm 0.027$ & $0.955 \pm 0.012$ & $0.985 \pm 0.009$ \\
\hline
\end{tabular}

Noted: Prec. $=$ Precision 

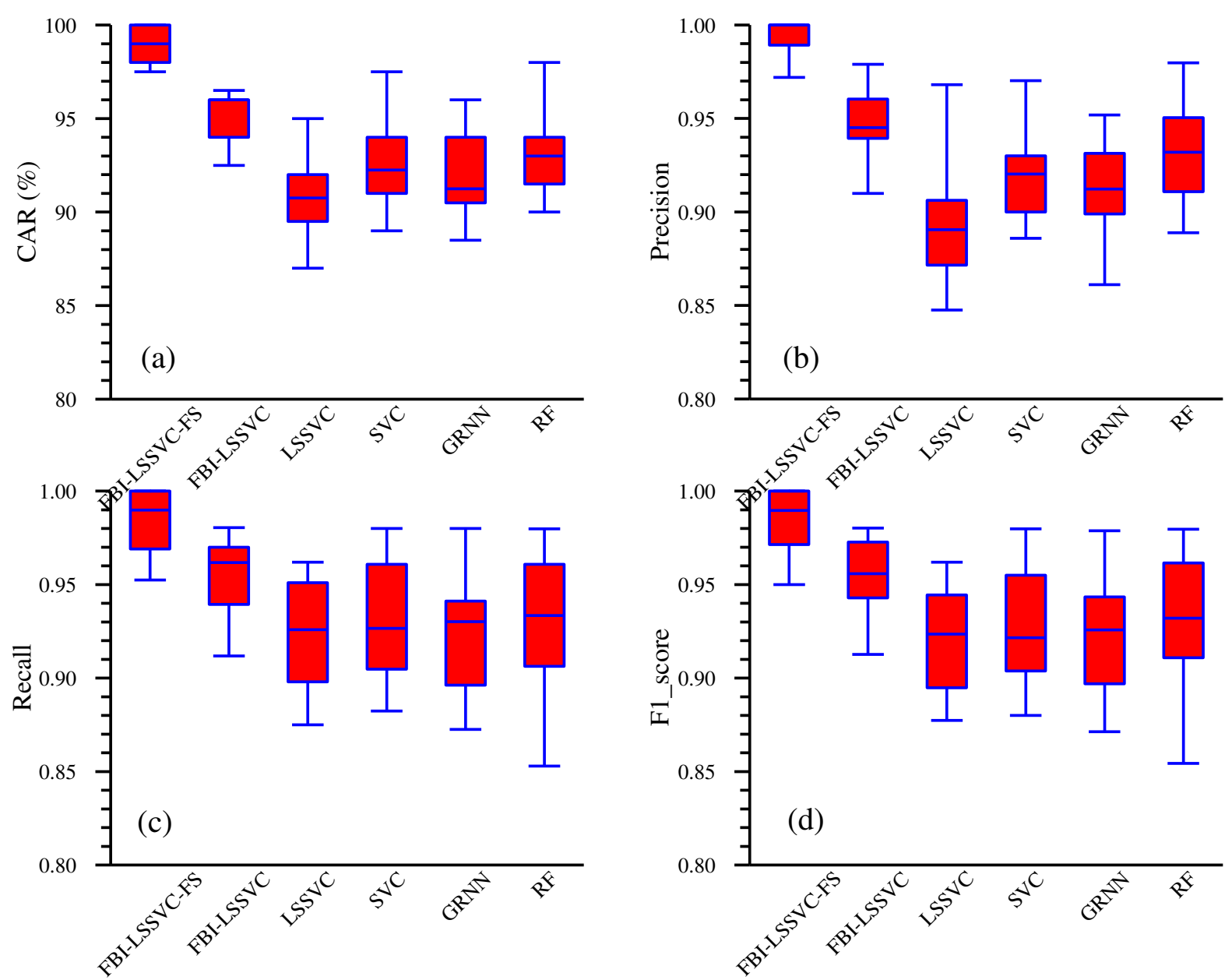

Fig. 8 Box-plot of comparative models 'performance: (a) CAR; (b) Precision; (c) Recall; (d) F1 score

Table 3 and Fig. 7 shows the results of all comparative models for identifying rutting damage of road on 20 run times. Apparently, all models were well trained with acquiring high values of CAR $(\geq 92 \%)$, precision $(\geq 0.909)$, recall $(\geq 0.934)$, F1_score $(\geq 0.921)$, and NPV ( $\geq 0.932)$. However, there are remarkable differences in terms of classification capability among the AI models in the validation phase. The proposed model, FBI-LSSVCFS is highlighted as the supreme model by gaining the greatest values for both mean and standard deviation of all performance indexes, particularly CAR (mean $=98.9 \%$; std. $=0.9 \%$ ), precision $($ mean $=0.994 ;$ std. $=0.008)$, recall $($ mean $=0.984 ;$ std. $=0.016)$, F1_score (mean $=0.989 ;$ std. $=0.016)$, and NPV (mean $=0.985 ;$ std. $=0.009)$. These values show that using the proposed model, road inspectors can achieve roughly $6.0 \%$ accuracy improvement which contributes a large portion of budget for allocating money to repair road damage. Noted that the attained low values of standard deviation support FBI-LSSVC-FS to have a stable performance; thus, the given classification outcomes are reliable.

As shown in Table 2 and Fig. 6, all of 94 features have been involved in the construction of classification models. However, selections for the set of features different for each run time which aims at synchronizing information for constructing model since there is a variance existing in each training dataset. The results of Table 2 and Table 3 further expose that the proposed model's performance has no bias in predicting any classes. Thus, the objective function is formulated correctly. Apparently, statistical results support the integration of FS and FBI in LSSVC to be rational for achieving the greatest accuracy of classifying rutting / non-rutting. Accordingly, FBI-LSSVC-FS accomplished the prediction 
test with $9.3 \%, 11.1 \%, 6.7 \%, 9.0 \%$, and $7.0 \%$ improvements compared with conventional LSSVC in terms of CAR, precision, recall, F1_score, and NPV, respectively. Undoubtedly, the exclusion of redundant features is essential to both reduce computational complexity of the inference model and disturbance information that may degrade prediction performance.

This study further employed a statistical method, named one-tailed $t$-test to verify the significant differences between comparative models' performance. The test is implemented on the CAR values yielded in the testing phase with equal samples as the following procedure:

- $H_{0}$ (null hypothesis) :CAR $F B I-L S S V C-F S-C A R_{\text {others }}=0$

- $H_{1}$ (reject hypothesis): CAR FBI-LSSVC-FS $-C A R_{\text {others }}>0$

Degree of freedom: $v=(n-1)\left(s_{1}^{2}+s_{2}^{2}\right)^{2} /\left(s_{1}^{4}+s_{2}^{4}\right)$

Statistical test: $t=\sqrt{n}\left(\bar{x}_{1}-\bar{x}_{2}\right) / \sqrt{s_{1}^{2}+s_{2}^{2}}$

where $n$ is number of run times $(\mathrm{n}=20) ; v$ is the degree of freedom; $s_{1}^{2}$ and $s_{2}^{2}$ are the unbiased estimators of the variances of the two samples; The denominator of $t$ is the standard error of the difference between two means $\bar{x}_{1}$ and $\bar{x}_{2}$.

Calculated results with a confidence level of 95\% $(\alpha=0.05)$ are presented in Table 4. For all cases, t-statistic > t-critical one-tailed (1.70), indicating FBI-LSSVC-FS significantly outperformed other models in increasing CAR values of classifying rutting / non-rutting. The conclusion may be reflected visually in Fig. 7.

Despite of possessing an outstanding inference performance, FBI-LSSVC-FS still gives mis-classification rutting / non-rutting of asphalt pavement. Fig. 8 displays several samples that FBI-LSSVC-FS incorrectly label images. Non-rutting is categorized into negative class as shown in Fig. 8a, b, c and d which caused by the fake trace of soil on the asphalt surface. Meanwhile, Fig. 8e, f, g, and h show samples of false positive. Surveyed and analyzed from the experiment, the mis-classification may be caused by a soil layer that fills the rutting and spread out the surrounding area (see Fig. 8g); thus, features of rutting are degenerated. Obtained features of a minor rutting are weak that also causes difficulty for the identification, as shown in Fig. 8e and f. Additionally, irregular patterns result in a negative impact on the model performance Fig. 8h. Nevertheless, this issue can be resolved by supplementing more images of the irregular patterns so that model can experience through the learning phase.

Table 4 Calculation of one-tailed $t$-test on CAR values

\begin{tabular}{lccccc}
\hline T-test & RF & GRNN & SVC & LSSVC & FBI-LSSVC \\
\hline$d f$ & 28 & 26 & 27 & 27 & 34 \\
\hline$t$-statistical & 12.71 & 13.76 & 13.62 & 17.26 & 10.37 \\
\hline $\mathrm{P}(\mathrm{T}<=\mathrm{t}) 1$-tail & $1.87 \mathrm{E}-13$ & $9.48 \mathrm{E}-14$ & $6.52 \mathrm{E}-14$ & $2.07 \mathrm{E}-16$ & $2.26 \mathrm{E}-12$ \\
\hline$t$-critical $l$-tail & 1.70 & 1.71 & 1.70 & 1.70 & 1.69 \\
\hline CONCLUSION & Reject HO & Reject HO & Reject $\mathrm{HO}$ & Reject $\mathrm{HO}$ & Reject $\mathrm{HO}$ \\
\hline
\end{tabular}


Samples of false negative (a, b, c, d)

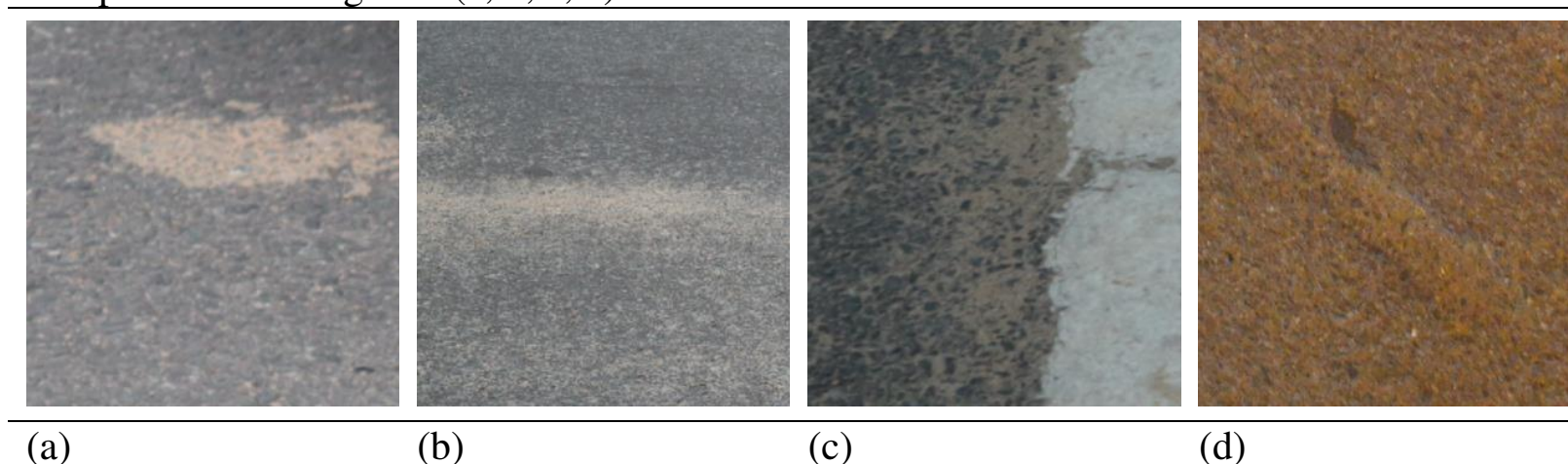

Samples of false positive (e, $\mathrm{f}, \mathrm{g}, \mathrm{h})$
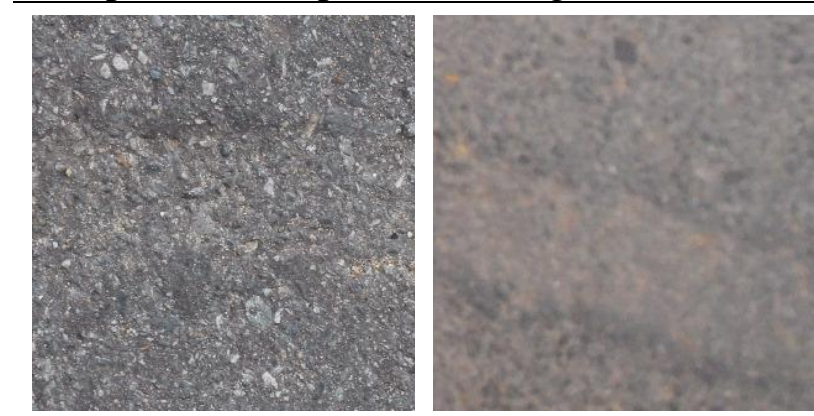

(f)
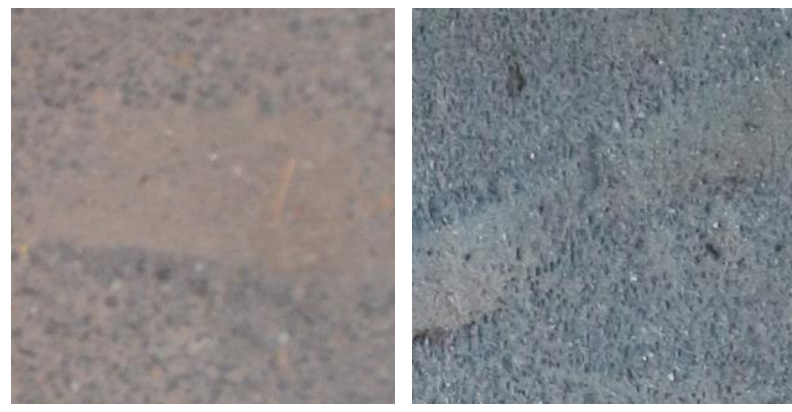

(e)

(g)

(h)

Fig. 9 Samples of misclassification: $(a, b, c, d)$ false negative; $(e, f, g, h)$ false positive 


\section{Conclusion}

Rutting presents a serious degradation of the asphalt pavements which is caused by heavy-vehicle wheel loads moving on poor compaction area or un-qualified asphalt layer of road. This degradation type thus needs to be early inspected for proceeding repairing task. This paper presents a novel hybrid machine learning based inference model to extract features of rutting for increasing prediction accuracy of rutting road. Texture of image is first analyzed and processed by GF and DCT techniques to generate potential features presenting rutting and non-rutting. The extracted features are then served as predictors for LSSVC model to build a hyperplane function of classifying images with rutting and non-rutting. This study further used FBI to search for optimal classification performance of LSSVC via a loop of optimization process which aims at determining set of hyper-parameters $(\mathrm{C}$ and $\gamma)$ and simultaneously refining set of highly relevant features to match with the hyperplane function of LSSVC.

The experiments have proven that all hybrid techniques fused in the proposed model have important roles in gaining the fascinating prediction accuracy. Statistical results point out that the proposed model, named as FBI-LSSVC-FS, achieved the most desirable values of CAR (98.9\%), precision (0.994), recall (0.984), and F1_score (0.989) which are significantly better than that of GRNN, RF, SVC. These obtained values further indicate that FBI and FS contributed an improvement of $9.3 \%$ and $11.1 \%$ in terms of CAR and precision, respectively. It is worth noting that none of 94 extracted features are assessed as redundant variables. However, roughly half of all features are filtered to include in establishing the LSSVC model in each run time due to the variance of information inherent in each of training datasets. The variance of information is processed by refining set of features that produces synchronizing information to promote the learning capability of LSSVC model. Undoubtedly, use of FBI to simultaneously determine values of hyper-parameters $(C$ and $\gamma)$ and dynamically assigned set of features corresponding to each training datasets is critical.

The analysis confirmed that the performance of prediction model is slightly compromised when working on images with strongly shadow texture, irregular patterns, or two gentle rutting. It is suggested to conduct the site trip survey at the sunny day and collect more image of irregular patterns or gentle rutting so that LSSVC has more chance of learning more deeply in the construction model phase. Hence, further steps of the current study include utilization of more advanced AI soft computing models and enriching image dataset to diminish effect of shadow and irregular pattern.

In addition to the strong power of prediction highlighted in the experiment and result analysis, the FBI-LSSVC-FS is able to work without human intervention because users are not required to finetune model's parameters. Hence, the FBI-LSSVC-FS is highly recommended to support transportation agencies in the surveying task. With the frame work of FBI-LSSVC-FS is believed to implement efficiently classification tasks for other type of road depression, such as cracking and pothole when it is trained with the corresponding image datasets. In summary, this paper contributes a novel method to the core body of knowledge for handling image classification task that is able to be extended as a commercial product.

\section{Supplementary Material}

The image data used in this study can be openly accessed at: https://github.com/NhatDucHoang/PavementRuttingData 


\section{Compliance with ethical standards}

\section{Conflict of interest statement}

All authors declare that they have no conflict of interest.

\section{Ethical approval}

This article does not contain any studies with human participants or animals performed by any of the authors.

\section{Informed consent}

Informed consent was obtained from all individual participants included in the study.

\section{Authorship contributions}

Conceptualization: Minh-Tu Cao, Kuan-Tsung Chang, Nhat-Duc Hoang.

Data collection and compilation: Nhat-Duc Hoang, Van-Duc Tran, Xuan-Linh Tran, NgocMai Nguyen.

Methodology: Kuan-Tsung Chang, Nhat-Duc Hoang, Minh-Tu Cao, Xuan-Linh Tran.

Writing - original draft preparation: Minh-Tu Cao, Nhat-Duc Hoang, Kuan-Tsung Chang, Ngoc-Mai Nguyen, Van-Duc Tran, Xuan-Linh Tran.

Writing - review and editing: Kuan-Tsung Chang, Nhat-Duc Hoang, Minh-Tu Cao.

\section{Reference}

Accord (2019) Accord.NET Framework http://accord-frameworknet/ (Last Access Date 09/08/2019)

Akagic A, Buza E, Omanovic S Pothole detection: An efficient vision based method using RGB color space image segmentation. In: 2017 40th International Convention on Information and Communication Technology, Electronics and Microelectronics (MIPRO), 22-26 May 2017 2017. pp 1104-1109. doi:10.23919/MIPRO.2017.7973589

An KE, Lee SW, Ryu S, Seo D Detecting a pothole using deep convolutional neural network models for an adaptive shock observing in a vehicle driving. In: 2018 IEEE International Conference on Consumer Electronics (ICCE), 12-14 Jan. 2018 2018. pp 1-2. doi:10.1109/ICCE.2018.8326142

Aparna, Bhatia Y, Rai R, Gupta V, Aggarwal N, Akula A (2019) Convolutional neural networks based potholes detection using thermal imaging Journal of King Saud University - Computer and Information Sciences doi:10.1016/j.jksuci.2019.02.004

Cheng M-Y, Prayogo D, Wu Y-W (2018) Prediction of permanent deformation in asphalt pavements using a novel symbiotic organisms search-least squares support vector regression Neural Computing and Applications doi:10.1007/s00521-018-3426-0

Chou J-S, Nguyen N-M (2020) FBI inspired meta-optimization Applied Soft Computing:106339 doi:https://doi.org/10.1016/j.asoc.2020.106339

Coenen TBJ, Golroo A (2017) A review on automated pavement distress detection methods Cogent Engineering 4 doi:10.1080/23311916.2017.1374822

Cubero-Fernandez A, Rodriguez-Lozano FJ, Villatoro R, Olivares J, Palomares JM (2017) Efficient pavement crack detection and classification EURASIP Journal on Image and Video Processing 2017:39 doi:10.1186/s13640-017-0187-0

Dorafshan S Comparing Automated Image-Based Crack Detection Techniques in Spatial and Frequency Domains. In: In Proc. of the 26th ASNT Research Symposium, at Jacksonville, Florida, 2017. pp 34-42

Dorafshan S, Thomas RJ, Maguire M (2018) Comparison of deep convolutional neural networks and edge detectors for image-based crack detection in concrete 
Construction and Building Materials 186:1031-1045

doi:10.1016/j.conbuildmat.2018.08.011

Douangphachanh V, Oneyama $\mathrm{H}$ (2014) A study on the use of smartphones under realistic settings to estimate road roughness condition EURASIP $\mathrm{J}$ Wireless Commun Network, 2014(1),114

Doycheva K, Koch C, König M Computer Vision and Deep Learning for Real-Time Pavement Distress Detection. In, Cham, 2019. Advances in Informatics and Computing in Civil and Construction Engineering. Springer International Publishing, pp 601-607

Eduardo Z, Jaime GGB, Roberto M, José L (2014) Road Crack Detection Using Visual Features Extracted by Gabor Filters Computer-Aided Civil and Infrastructure Engineering 29:342-358 doi:doi:10.1111/mice.12042

Gao M, Wang X, Zhu S, Guan P (2020) Detection and Segmentation of Cement Concrete Pavement Pothole Based on Image Processing Technology Mathematical Problems in Engineering Volume 2020 |Article ID 1360832 | 13 pages https://doi.org/10.1155/2020/1360832

Georgiou P, Plati C, Loizos A (2018) Soft Computing Models to Predict Pavement Roughness: A Comparative Study Advances in Civil Engineering 2018:8 doi:10.1155/2018/5939806

Gopalakrishnan K (2018) Deep Learning in Data-Driven Pavement Image Analysis and Automated Distress Detection: A Review Data 3:28

Gopalakrishnan K, Khaitan SK, Choudhary A, Agrawal A (2017) Deep Convolutional Neural Networks with transfer learning for computer vision-based data-driven pavement distress detection Construction and Building Materials 157:322-330 doi:https://doi.org/10.1016/j.conbuildmat.2017.09.110

Guan H, Li J, Yu Y, Chapman M, Wang H, Wang C, Zhai R (2015) Iterative Tensor Voting for Pavement Crack Extraction Using Mobile Laser Scanning Data IEEE Transactions on Geoscience and Remote Sensing 53:1527-1537 doi:10.1109/TGRS.2014.2344714

Hadjidemetriou GM, Christodoulou SE, Vela PA (2016) Automated detection of pavement patches utilizing support vector machine classification In Proc of the 18th Mediterranean Electrotechnical Conf (MELECON), IEEE, Piscataway, NJ, 1-5

Hadjidemetriou GM, Masino J, Christodoulou SE, Gauterin F, Brilakis I (2020) Comprehensive Decision Support System for Managing Asphalt Pavements Journal of Transportation Engineering, Part B: Pavements 146:06020001 doi:doi:10.1061/JPEODX.0000189

Hadjidemetriou GM, Vela PA, Christodoulou SE (2018) Automated Pavement Patch Detection and Quantification Using Support Vector Machines Journal of Computing in Civil Engineering 32:04017073 doi:10.1061/(ASCE)CP.1943-5487.0000724

Hammouda K (2000) Texture Segmentation Using Gabor Filters Technical Report, University of Waterloo, http://wwwpamiuwaterlooca/pub/hammouda/sd775-paperpdf (Last Access Date 09/20/2019)

Hee-Jung B, Sung-Hwan J Image retrieval using texture based on DCT. In: Proceedings of ICICS, 1997 International Conference on Information, Communications and Signal Processing. Theme: Trends in Information Systems Engineering and Wireless Multimedia Communications (Cat., 12-12 Sept. 1997 1997. pp 1065-1068 vol.1062. doi:10.1109/ICICS.1997.652144

Hoang N-D (2018) An Artificial Intelligence Method for Asphalt Pavement Pothole Detection Using Least Squares Support Vector Machine and Neural Network with Steerable Filter-Based Feature Extraction Advances in Civil Engineering:1-12 doi:10.1155/2018/7419058

Hoang N-D (2019a) Automatic detection of asphalt pavement raveling using image texture based feature extraction and stochastic gradient descent logistic regression Automation in Construction

doi:https://doi.org/10.1016/j.autcon.2019.102843

105:102843 
Hoang N-D (2019b) Image processing based automatic recognition of asphalt pavement patch using a metaheuristic optimized machine learning approach Advanced Engineering Informatics 40:110-120 doi:https://doi.org/10.1016/j.aei.2019.04.004

Hoang N-D, Nguyen Q-L (2018) Fast Local Laplacian-Based Steerable and Sobel Filters Integrated with Adaptive Boosting Classification Tree for Automatic Recognition of Asphalt Pavement Cracks Advances in Civil Engineering 2018:17 doi:10.1155/2018/5989246

Hoang N-D, Nguyen Q-L, Bui DT (2018a) Image Processing-Based Classification of Asphalt Pavement Cracks Using Support Vector Machine Optimized by Artificial Bee Colony Journal of Computing in Civil Engineering 32:04018037 doi:doi:10.1061/(ASCE)CP.1943-5487.0000781

Hoang N-D, Nguyen Q-L, Tran V-D (2018b) Automatic recognition of asphalt pavement cracks using metaheuristic optimized edge detection algorithms and convolution neural network Automation in Construction 94:203-213 doi:10.1016/j.autcon.2018.07.008

Jain AK, Farrokhnia F (1991) Unsupervised texture segmentation using Gabor filters Pattern Recognition 24:1167-1186 doi:https://doi.org/10.1016/0031-3203(91)90143-S

Jo J, Jadidi Z (2019) A high precision crack classification system using multi-layered image processing and deep belief learning Structure and Infrastructure Engineering:1-9 doi:10.1080/15732479.2019.1655068

Joanes DN, Gill CA (1998) Comparing measures of sample skewness and kurtosis Journal of the Royal Statistical Society: Series D (The Statistician) 47:183-189 doi:10.1111/1467-9884.00122

Kachouie NN, Alirezaie J, Fieguth P A hybrid algorithm using discrete cosine transform and Gabor filter bank for texture segmentation. In: Canadian Conference on Electrical and Computer Engineering 2004 (IEEE Cat. No.04CH37513), 2-5 May 20042004. pp 1805-1808 Vol.1803. doi:10.1109/CCECE.2004.1349767

Kaddah W, Elbouz M, Ouerhani Y, Baltazart V, Desthieux M, Alfalou A (2018) Optimized minimal path selection (OMPS) method for automatic and unsupervised crack segmentation within two-dimensional pavement images The Visual Computer doi:10.1007/s00371-018-1515-9

Kim NC, So HJ (2018) Directional statistical Gabor features for texture classification Pattern Recognition Letters 112:18-26 doi:https://doi.org/10.1016/j.patrec.2018.05.010

Li S, Cao Y, Cai H (2017) Automatic Pavement-Crack Detection and Segmentation Based on Steerable Matched Filtering and an Active Contour Model Journal of Computing in Civil Engineering 31:04017045 doi:doi:10.1061/(ASCE)CP.1943-5487.0000695

Liley C (2018) Rutting: Causes, Prevention, and Repairs Illinois Asphalt Pavement Association, https://wwwilasphaltorg/files/9915/1820/9831/Chance_Liley_2017_SIUEpdf (Last Access Date 11/07/2019)

Liu P, Otto F, Wang D, Oeser M, Balck H (2017) Measurement and evaluation on deterioration of asphalt pavements by geophones Measurement 109:223-232 doi:https://doi.org/10.1016/j.measurement.2017.05.066

Massahi A, Ali H, Koohifar F, Baqersad M, Mohammadafzali M (2018) Investigation of pavement raveling performance using smartphone International Journal of Pavement Research and Technology 11:553-563 doi:10.1016/j.jprt.2017.11.007

Mathavan S, Rahman MM, Stonecliffe-Jones M, Kamal K (2014) Pavement Raveling Detection and Measurement from Synchronized Intensity and Range Images Transportation Research Record 2457:3-11 doi:10.3141/2457-01

Medina R, Gayubo F, González-Rodrigo LM, Olmedo D, Gómez-García-Bermejo J, Zalama E, Perán JR (2011) Automated visual classification of frequent defects in flat steel coils The International Journal of Advanced Manufacturing Technology 57:1087-1097 doi:10.1007/s00170-011-3352-0

Orenstein T, Kohavi Z, Pomeranz I (1995) An optimal algorithm for cycle breaking in directed graphs J Electron Test 7:71-81 doi:10.1007/BF00993315 
Ouma YO, Hahn M (2016) Wavelet-morphology based detection of incipient linear cracks in asphalt pavements from RGB camera imagery and classification using circular Radon transform Advanced Engineering Informatics 30:481-499 doi:https://doi.org/10.1016/j.aei.2016.06.003

Ouma YO, Hahn M (2017) Pothole detection on asphalt pavements from 2D-colour pothole images using fuzzy c-means clustering and morphological reconstruction Automation in Construction 83:196-211 doi:https://doi.org/10.1016/j.autcon.2017.08.017

Pun C-M, Zhu H-M (2009) Textural image segmentation using discrete cosine transform. Paper presented at the Proceedings of the 3rd International Conference on Communications and information technology, Vouliagmeni, Athens, Greece,

Radopoulou SC, Brilakis I (2015) Patch detection for pavement assessment Automation in Construction 53:95-104 doi:10.1016/j.autcon.2015.03.010

Salman M, Mathavan S, Kamal K, Rahman M Pavement crack detection using the Gabor filter. In: 16th International IEEE Conference on Intelligent Transportation Systems (ITSC 2013), 6-9 Oct. 2013 2013. pp 2039-2044. doi:10.1109/ITSC.2013.6728529

Subudhi P, Mukhopadhyay S (2017) A fast texture segmentation scheme based on active contours and discrete cosine transform Computers \& Electrical Engineering 62:105118 doi:https://doi.org/10.1016/j.compeleceng.2017.04.021

Suykens J, Gestel JV, Brabanter JD, Moor BD, Vandewalle J (2002) Least Squares Support Vector Machines. World Scientific Publishing Co. Pte. Ltd., ISBN-13: 9789812381514,

Tsai T, Huang Y, Chiang T Image Retrieval Based on Dominant Texture Features. In: 2006 IEEE International Symposium on Industrial Electronics, 9-13 July 2006 2006. pp 441-446. doi:10.1109/ISIE.2006.295635

Wu Y-H, Shen H (2018) Grey-related least squares support vector machine optimization model and its application in predicting natural gas consumption demand Journal of Computational and Applied Mathematics 338:212-220 doi:https://doi.org/10.1016/j.cam.2018.01.033

Yao X, Yao M, Xu B Automated Detection and Identification of Area-based Distress in Concrete Pavements. In: Seventh International Conference on Managing Pavement Assets, Calgary Alberta, Canada, 2008-6-23 to 2008-6-28 2008. The National Academies of Sciences, Engineering, and Medicine,

Yousaf MH, Azhar K, Murtaza F, Hussain F (2018) Visual analysis of asphalt pavement for detection and localization of potholes Advanced Engineering Informatics 38:527-537 doi:10.1016/j.aei.2018.09.002

Zakeri H, Nejad FM, Fahimifar A (2017) Image Based Techniques for Crack Detection, Classification and Quantification in Asphalt Pavement: A Review Archives of Computational Methods in Engineering 24:935-977 doi:10.1007/s11831-016-9194-z

Zehang S, Bebis G, Miller R (2005) On-road vehicle detection using evolutionary Gabor filter optimization IEEE Transactions on Intelligent Transportation Systems 6:125-137 doi:10.1109/TITS.2005.848363

Zhang A et al. (2018a) Deep Learning-Based Fully Automated Pavement Crack Detection on 3D Asphalt Surfaces with an Improved CrackNet Journal of Computing in Civil Engineering 32:04018041 doi:doi:10.1061/(ASCE)CP.1943-5487.0000775

Zhang K, Cheng HD, Zhang B (2018b) Unified Approach to Pavement Crack and Sealed Crack Detection Using Preclassification Based on Transfer Learning Journal of Computing in Civil Engineering 32:04018001 doi:10.1061/(ASCE)CP.19435487.0000736 


\section{Figures}

\begin{tabular}{|l|l|l|l|l|l|l|l|}
\hline$C(0,0)$ & $C(0,1)$ & $C(0,2)$ & $C(0,3)$ & $C(0,4)$ & $C(0,5)$ & & \\
\hline$C(1,0)$ & $C(1,1)$ & $C(1,2)$ & $C(1,3)$ & $C(1,4)$ & $C(1,5)$ & & \\
\hline$C(2,0)$ & $C(2,1)$ & $C(2,2)$ & $C(2,3)$ & & & & \\
\hline$C(3,0)$ & $C(3,1)$ & $C(3,2)$ & $C(3,3)$ & $C(3,4)$ & & & \\
\hline$C(4,0)$ & $C(4,1)$ & & $C(4,3)$ & $C(4,4)$ & $C(4,5)$ & & \\
\hline$C(5,0)$ & $C(5,1)$ & & & $C(5,4)$ & $C(5,5)$ & & \\
\hline & & & & & & & \\
\hline & & & & & & & \\
\hline
\end{tabular}

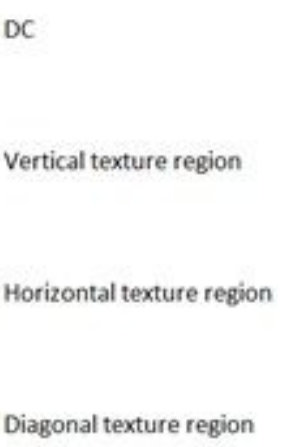

Figure 1

The extracted DCT coefficients

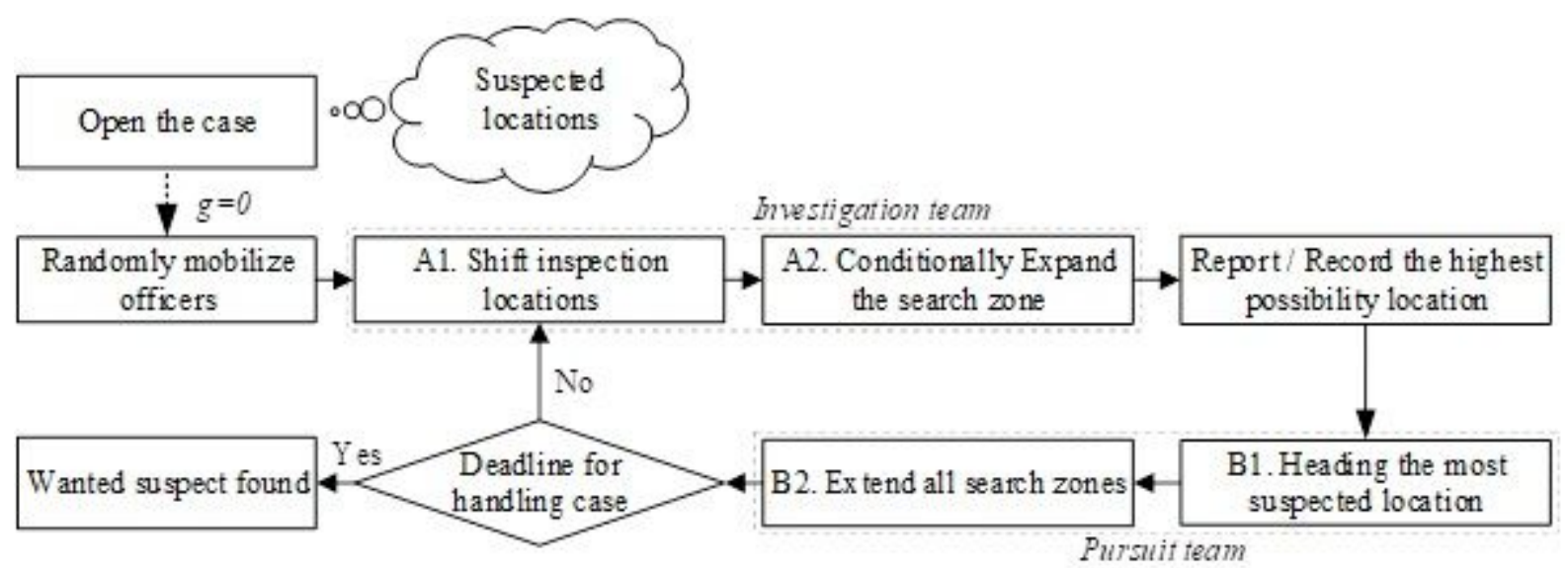

Figure 2

Flowchart of the $\mathrm{FBI}$ algorithm
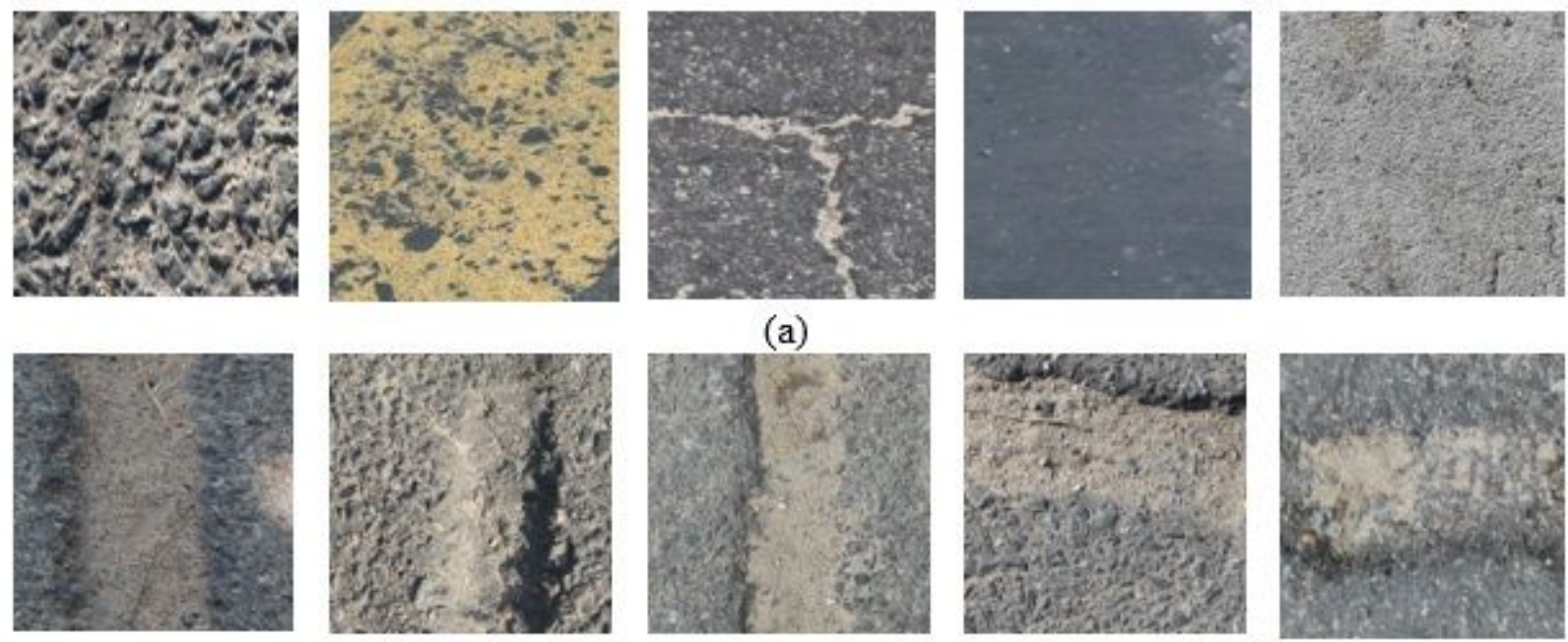

(a)
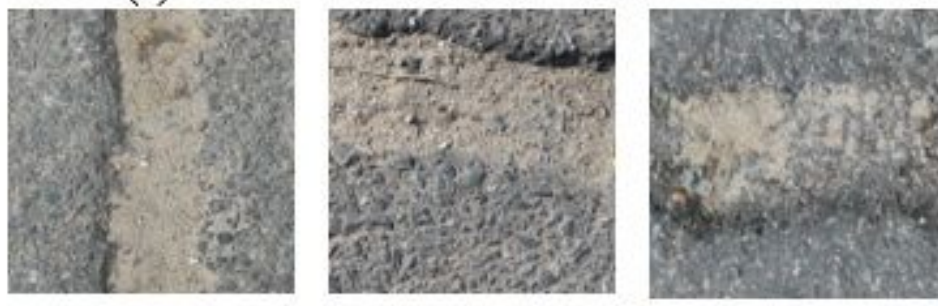

(1.) 


\section{Figure 3}

Illustration of the collected pavement images: (a) Non-rutting class and (b) Rutting class

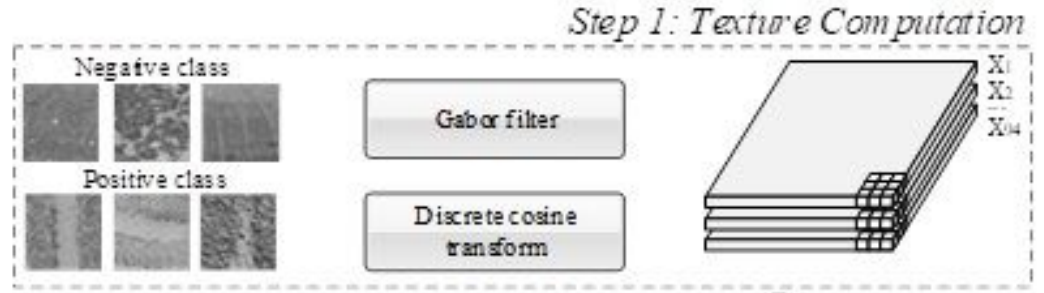

ת Step2: FBI-LSSVC-FS
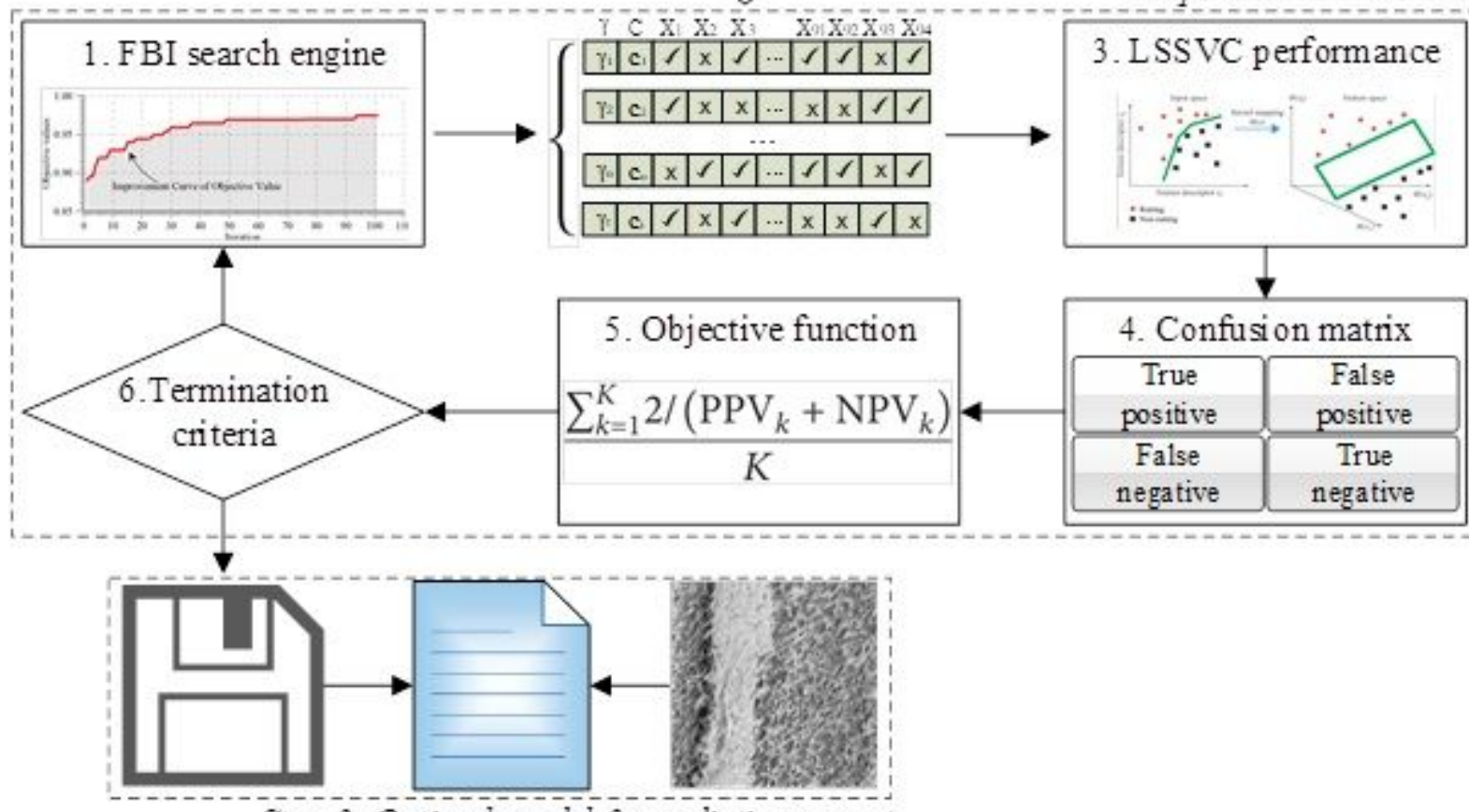

Step 3: Optim al model \& prediction report

Figure 4

The proposed FBI-LSSVC-FS Model Structure

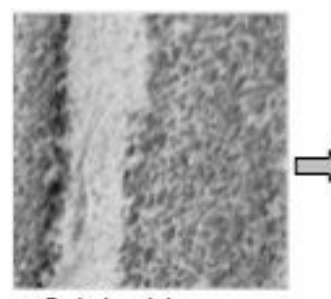

Original im age

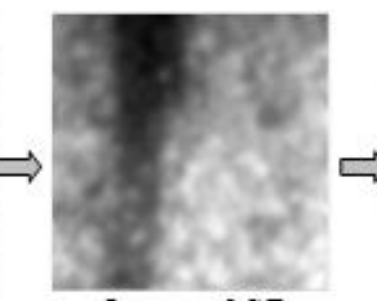

Output of GF

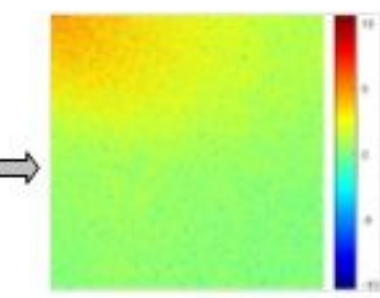

Output of DCT

$\Omega$

\begin{tabular}{|c|c|c|c|c|c|c|}
\hline$X_{1}$ & $\mathrm{X}_{2}$ & $\mathrm{X}$ & $\mathrm{X}_{4}$ & $\ldots$ & $\mathrm{X}_{93}$ & $\mathrm{X}_{94}$ \\
\hline 2218.39 & 7.80 & 9.19 & -11.26 & $\ldots$ & -36.04 & 507 \\
\hline
\end{tabular}

\section{Figure 5}

Illustration of the feature extraction process 


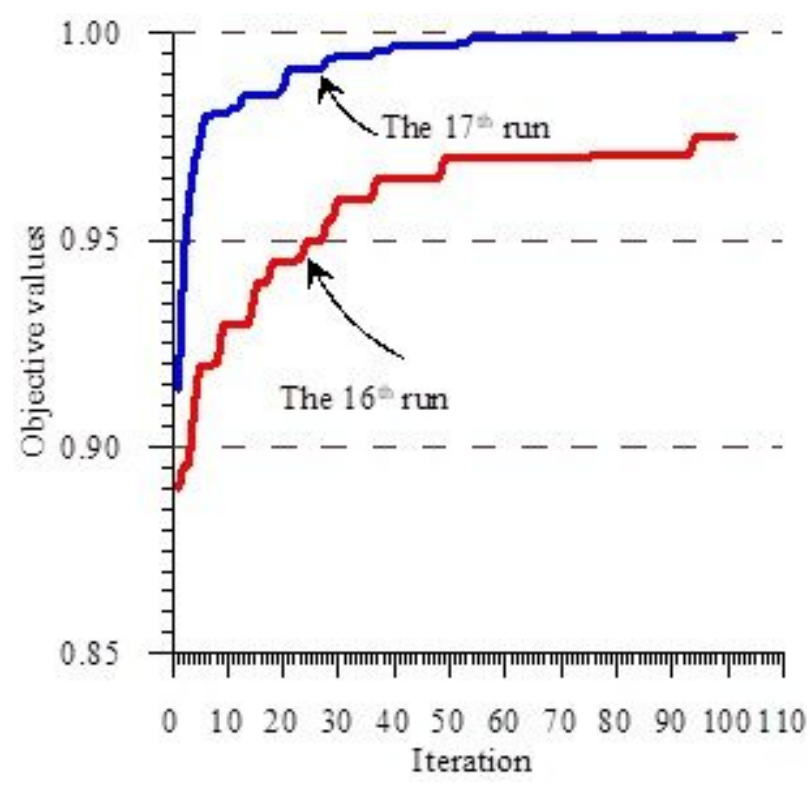

Figure 6

Improvement of objective values

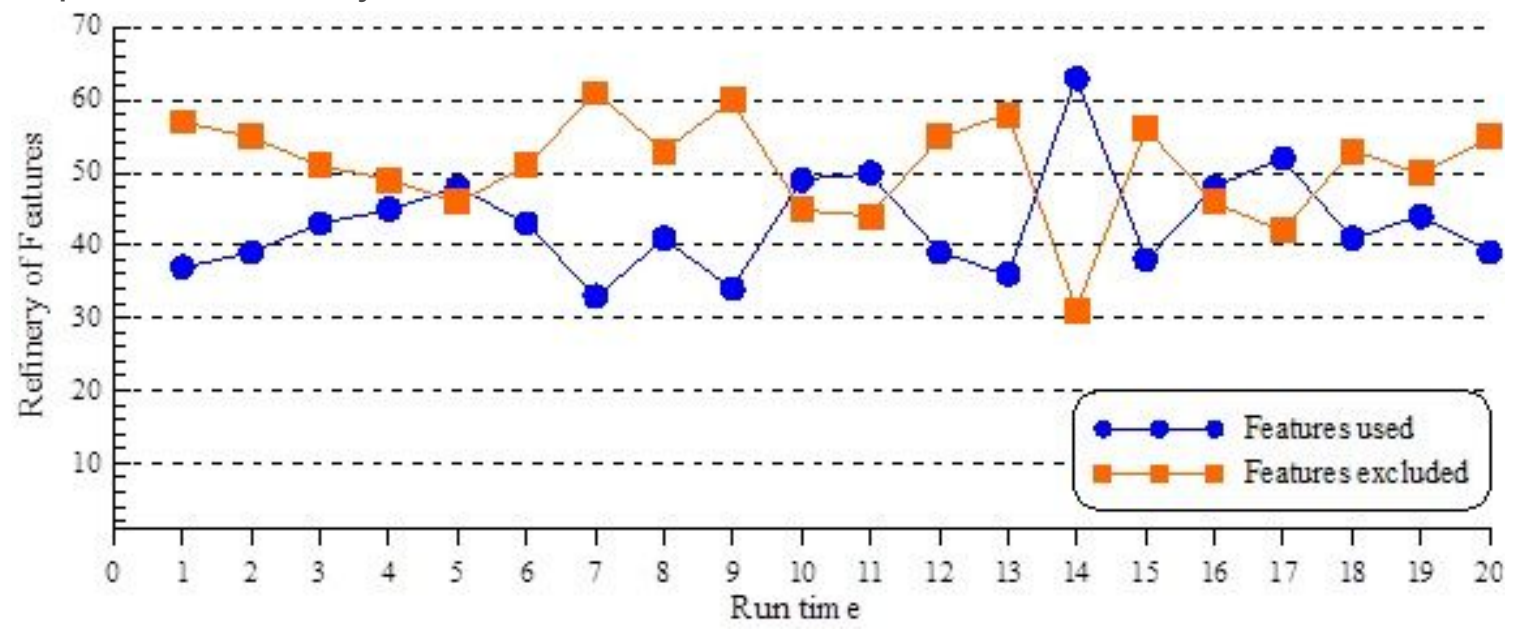

Figure 7

Features refined for LSSVC model construction 


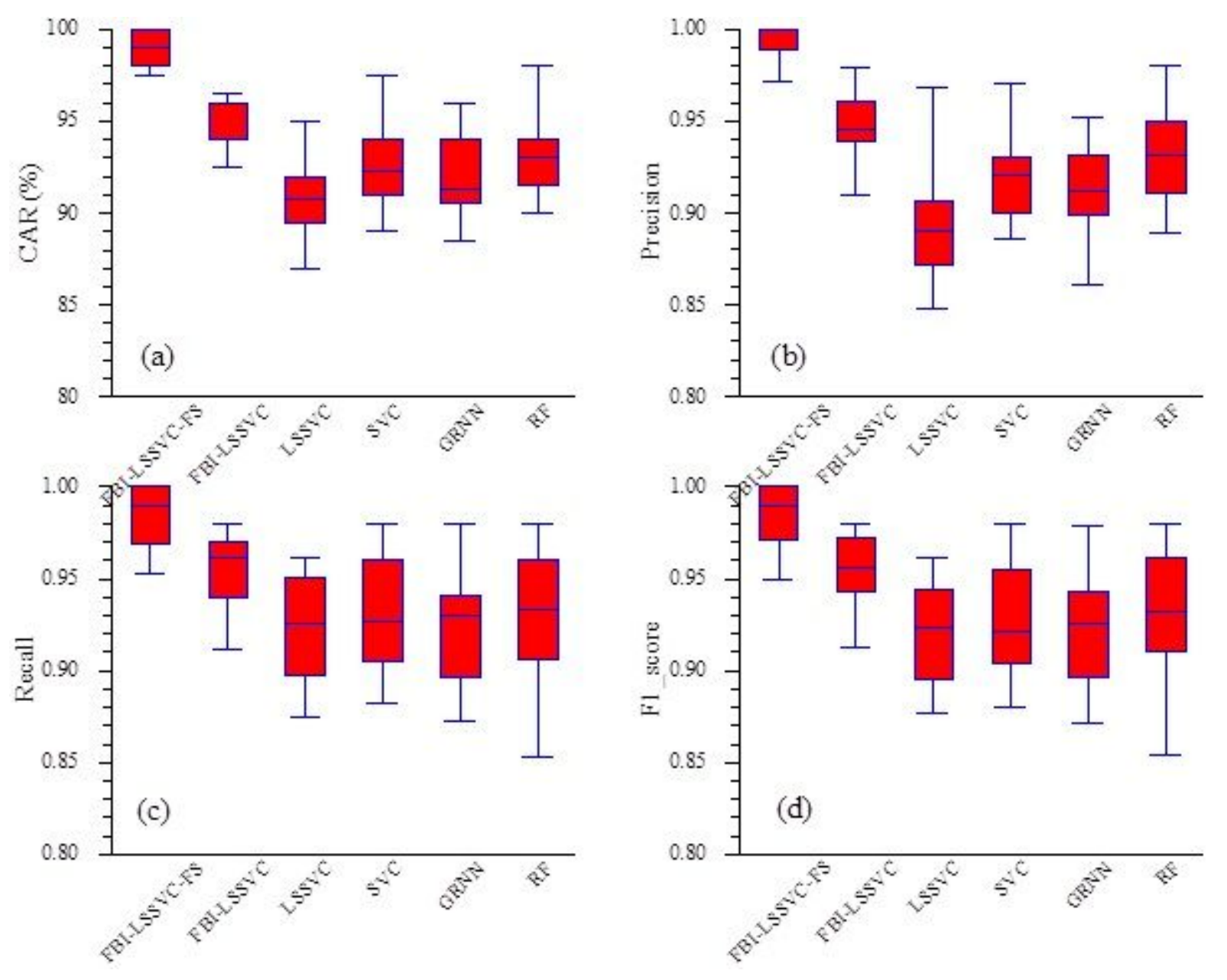

Figure 8

Box-plot of comparative models 'performance: (a) CAR; (b) Precision; (c) Recall; (d) F1 score 

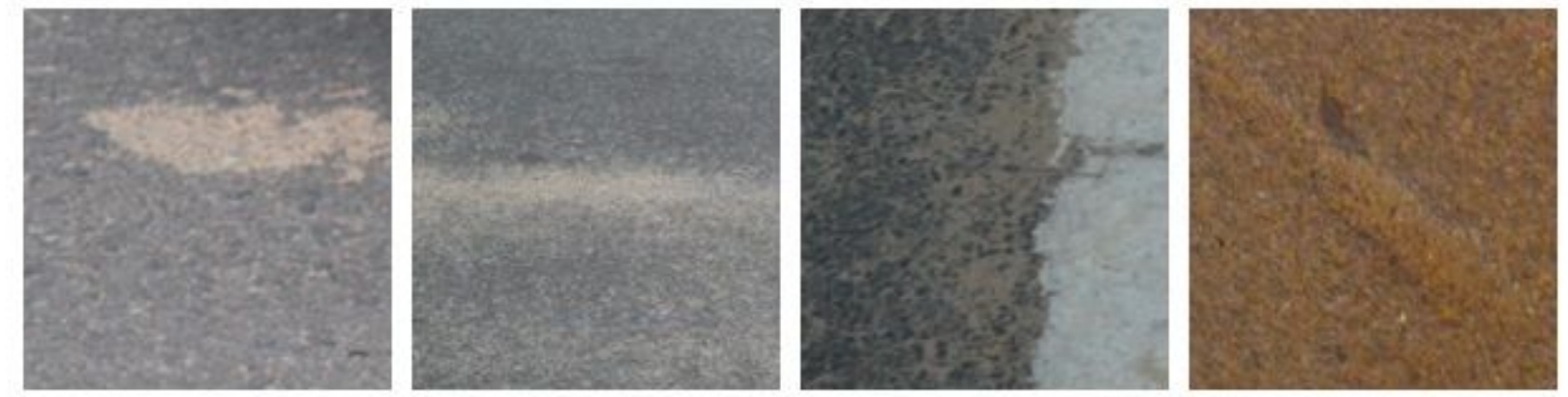

(a)

(b)

(c)

(d)

Samples of false positive $(e, f, g, h)$
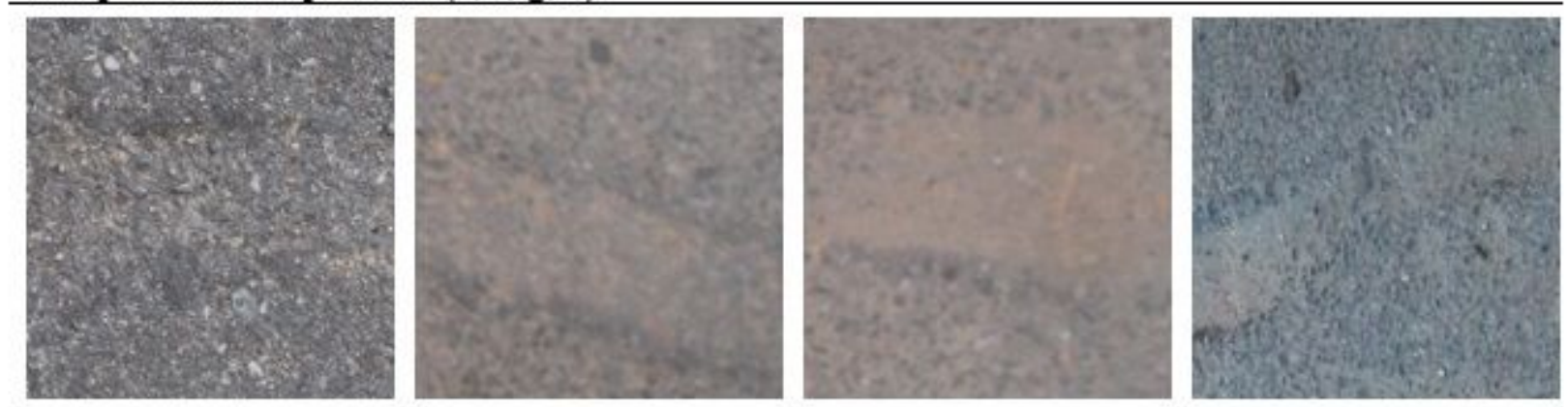

(e)

(f)

(g)

(h)

Figure 9

Samples of misclassification: $(a, b, c, d)$ false negative; $(e, f, g, h)$ false positive 